\title{
NONCOMMUTATIVE RIEMANNIAN AND SPIN GEOMETRY OF THE STANDARD $q$-SPHERE
}

\author{
S. MAJID
}

\begin{abstract}
We study the quantum sphere $\mathbb{C}_{q}\left[S^{2}\right]$ as a quantum Riemannian manifold in the quantum frame bundle approach. We exhibit its 2-dimensional cotangent bundle as a direct sum $\Omega^{0,1} \oplus \Omega^{1,0}$ in a double complex. We find the natural metric, volume form, Hodge * operator, Laplace and Maxwell operators and projective module structure. We show that the $q$-monopole as spin connection induces a natural Levi-Civita type connection and find its Ricci curvature and $q$-Dirac operator $\not \nabla$. We find the possibility of an antisymmetric volume form quantum correction to the Ricci curvature and Lichnerowicztype formulae for $\not^{2}$. We also remark on the geometric $q$-Borel-Weil-Bott construction.
\end{abstract}

\section{INTRODUCTION}

The standard quantum sphere is nothing other than the invariant subalgebra of the standard quantum group coordinate ring $\mathbb{C}_{q}\left[S L_{2}\right]$ under a coaction of $\mathbb{C}\left[t, t^{-1}\right]$. In the *-algebra setting it means $S U_{2} / U(1)$ in a coordinate form and of course $q$-deformed. Other nonstandard quantum spheres were constructed and classified in Po1 while a unique left-covariant 2-dimensional differential calculus on the standard $\mathbb{C}_{q}\left[S^{2}\right]$ was found in $[\mathrm{Po} 2, \mathrm{Po}]$. Meanwhile, the $q$-monopole principal bundle with total space $\mathbb{C}_{q}\left[S L_{2}\right]$ and base $\mathbb{C}_{q}\left[S^{2}\right]$ (i.e. the Hopf fibration) was constructed as an example of the theory of quantum principal bundles in BM1] and has been somewhat studied since, so that many of the ingredients of geometry for $\mathbb{C}_{q}\left[S^{2}\right]$ are already known.

In this paper we extend this geometry of $\mathbb{C}_{q}\left[S^{2}\right]$ to include Riemannian structures and a geometrically natural Dirac operator, using a systematic frame bundle approach to noncommutative geometry in [M1, M2, M3. In fact the point is not just to obtain good geometrically justified proposals for these structures on the algebra $\mathbb{C}_{q}\left[S^{2}\right]$ in isolation, but rather to demonstrate that the frame bundle formulation, which is formulated in principle at the level of any unital algebra, indeed includes such an important example and gives reasonable answers for it. This is important because without the straight-jacket of a general theory that applies across diverse examples (including ones not related to $q$-deformations) one could not have confidence that a given definition was not ad-hoc, without which one could not attach weight to physical or other predictions. We find that $\mathbb{C}_{q}\left[S^{2}\right]$ indeed fits perfectly into this quantum frame bundle approach to noncommutative geometry as a 'quantum framed Riemannian manifold'. Another motivation comes from the operator-algebras and K-theory approach to noncommutative geometry of Connes and others $[\mathrm{Co}]$. How this can be reconciled with quantum groups is an active

Date: July 2003/Rev. 1.

S.M. is a Royal Society University Research Fellow. 
and important area of research at the moment. While we proceed only from the quantum groups side, by going up to the point of the $q$-geometrically natural Dirac operator and $q$-spin bundle, one can begin to compare with the 'top down' Connes approach where an axiomatically defined 'Dirac' operator implicitly defines the geometry. We find for example that our $\not$ indeed generates the exterior derivative by commutator, as it should (equation (30)).

The principal features of the geometry on $\mathbb{C}_{q}\left[S^{2}\right]$ that we find are as follows. The most important feature is that, unlike previous examples based on quantum groups, the sphere is not parallelizable. Hence there is no global 'vielbein' and one must work 'upstairs' on the total space of the frame bundle for global formulae. Unlike usual formulae in physics, in the noncommutative case we do not consider coordinate charts or patching transformations, instead we use only global constructions. Here $\mathbb{C}_{q}\left[S^{2}\right]$ is the simplest example with this difficulty and hence a good setting in which to demonstrate that the frame bundle theory works. In fact we show, Theorem 2.1, that any quantum homogeneous space induced by a Hopf algebra surjection is a framed quantum manifold (we construct the soldering form). The construction at the level of universal calculi was in [M1 but we extend this to general differential calculi as needed for our example. To be self-contained, Section 2 starts by recalling the required quantum homogeneous bundle construction itself (before using it as frame bundle).

The rest of the paper computes what the general frame bundle approach implies for the particular example of $\mathbb{C}_{q}\left[S^{2}\right]$. Here for quantum frame bundle we take the quantum Hopf fibration or ' $q$-monopole principal bundle' from BM1, BM2, in an appropriate form. The role of the fiber $S O(2)$ frame rotations is played by the commutative Hopf algebra $\mathbb{C}\left[t, t^{-1}\right]$ equipped with a noncommutative $q$-deformation of its usual calculus. We then find the cotangent bundle $\Omega^{1}\left(\mathbb{C}_{q}\left[S^{2}\right]\right)$ as an associated bundle to this. This means that, like all associated bundles in this context (when there is a connection), the cotangent bundle is necessarily projective, a point of view in keeping with other approaches such as $\mathrm{Co}$. We later (in Section 5) exhibit its nontrivial projector explicitly. This is in spite of the fact that we find (Theorem 3.1) that the cotangent bundle is the sum of a charge -2 and charge 2 monopole, which means that is is zero in the (noncommutative) K-theory of $\mathbb{C}_{q}\left[S^{2}\right]$ (this is actually in keeping with the classical geometry). More importantly for us, Theorem 3.1 implies a natural direct sum decomposition of the cotangent bundle into much simpler "holomorphic" and "antiholomorphic" parts $\Omega^{1}\left(\mathbb{C}_{q}\left[S^{2}\right]\right)=\Omega^{0,1} \oplus \Omega^{1,0}$ according to the monopole charge, which we then use extensively in the sequel.

Section 4 covers the next 'layer' of geometry in the form of the exterior algebra, metric, Hodge * operator, Laplace operator and Maxwell theory. In particular, the natural $\mathbb{C}_{q}\left[S L_{2}\right]$-covariant metric $g$, Hodge $*$ and volume 2 -form (or symplectic structure) $\Upsilon$ lifted to an element $i(\Upsilon) \in \Omega^{1} \bar{\otimes} \Omega^{1}$ are naturally related as $(\mathrm{id} \bar{\otimes} *)(g) \propto$ $i(\Upsilon)$ (Proposition 4.3).

Section 5 then comes to the Riemannian geometry and contains our main result (Theorem 5.1) that the $q$-monopole as spin connection on the frame bundle indeed induces the correct generalized Levi-Civita connection $\nabla$ on the cotangent bundle for its natural metric. This is torsion free and 'cotorsion free'. The latter is a natural formulation of metric compatibility in the skew form

$$
(\nabla \wedge \mathrm{id}-\mathrm{id} \wedge \nabla) g=0
$$


This condition was proposed in the axioms of [M1, M2] for 'quantum Riemannian manifolds' as a weakening of usual metric compatibility suggested by noncommutative geometry. We see that $\mathbb{C}_{q}\left[S^{2}\right]$ indeed bears this out. We also compute the Riemann curvature of $\nabla$ as a 2-form-valued operator on 1-forms, and from this the physically all-important Ricci curvature. There is some freedom in the definition of this involved in choosing the lifting map $i$ but we find that with the natural $i(\Upsilon)$ modified by the addition of a $q$-symmetric metric term, one has Ricci proportional to the metric. Thus $\mathbb{C}_{q}\left[S^{2}\right]$ can be made into an 'Einstein space' (Proposition 5.2). Alternatively, we could not modify $i(\Upsilon)$, in which case we find

$$
\text { Ricci }=\frac{q^{-1}\left(1+q^{4}\right)}{2} g+\frac{[2]_{q}\left(1-q^{4}\right)}{2} i(\Upsilon)
$$

showing a quantum correction involving the $q$-antisymmetric volume form or symplectic structure. This effect could also be formulated as a $q$-antisymmetric addition to the metric itself, which would be in keeping with ideas from string theory, for example. While the correct physical point of view and consequent predictions of $q$-modifications to gravity would need an understanding of the noncommutative stress energy tensor (which will be attempted elsewhere), we see the possibility of a new physical effect that vanishes as $q \rightarrow 1$.

Finally, for spin bundle we take $\mathcal{S}=\mathcal{S}_{-} \oplus \mathcal{S}_{+}$the direct sum of the charge -1 and charge $1 q$-monopole bundles. This is then the correct 'double cover' of the cotangent bundle in terms of the corepresentation of $\mathbb{C}\left[t, t^{-1}\right]$. The same $q$ monopole connection on the frame bundle as used for $\nabla$ now induces a covariant derivative $D$ on $\mathcal{S}$. This combines with a natural $\mathbb{C}_{q}\left[S L_{2}\right]$-covariant $\gamma$-matrix which we provide, to give our gravitational Dirac operator $\not$. It has the correct $\mathbb{Z}_{2}$-graded form and we show (Proposition 5.5 and equation (29)) that its square is linked to the scalar Laplacian. It is also relatively computable. For example,

$$
\left(\begin{array}{c} 
\pm q^{\frac{1}{2}} a \\
b
\end{array}\right), \quad\left(\begin{array}{c} 
\pm q^{\frac{1}{2}} c \\
d
\end{array}\right)
$$

are eigenspinors of mass $\pm q^{\frac{1}{2}}$, where $a, b, c, d$ are the usual quantum group $\mathbb{C}_{q}\left[S L_{2}\right]$ generators, viewed now as spinor components. We work algebraically and do not provide Hilbert space or other analytic structures; this would need further study. We do, however, show that unlike the cotangent bundle, our spinor bundle is trivial and we exhibit its trivialisation $\mathcal{S} \cong \mathbb{C}_{q}\left[S^{2}\right] \oplus \mathbb{C}_{q}\left[S^{2}\right]$. Our $\not \nabla$ in the trivialisation appears to be more complicated than previous attempts at the Dirac operator on the $q$-sphere such as $[\mathrm{PS}$, but comes with the full geometrical picture above.

The appendix applies the $q$-monopole connection to formulate the $q$-Borel-WeilBott construction as a byproduct of the $q$-geometry in the paper. The generalisation of this to other quantum groups and of the Riemannian and spin $q$-geometry to other $q$-symmetric spaces are two directions for further work. Notably for physics, a suitable $\mathbb{C}_{q}\left[S^{4}\right]$ and $q$-instanton are known[BCT] but require the more general coalgebra bundle theory for which nonuniversal differential calculi are not yet formulated.

Acknoweldgements. I would like to thank Ruibin Zhang for stimulating discussions on the Borel-Weil-Bott construction (see appendix) during a visit to the Dept of Mathematics, University of Sydney in December 2002. 
Preliminaries. We take $\mathbb{C}_{q}\left[S L_{2}\right]$ in the conventions of, for example, the text $\left.\mathrm{Ma}\right]$. Namely it has a matrix $\left(\begin{array}{ll}a & b \\ c & d\end{array}\right)$ of generators with $b a=q a b$ etc. These are the 'lexicographical conventions' whereby $q$ is needed to put things in lexicographical order. We will frequently use the $q$-determinant relations $a d=1+q^{-1} b c$ and $d a=1+q b c$. The Hopf algebra structure has the usual matrix coproduct $\Delta$ and counit $\epsilon$ on the generators and the antipode or 'linearised inverse' is $S a=d, S d=a$, $S b=-q b, S c=-q^{-1} c$. For the axioms of Hopf algebra and basic notions such as actions and coactions, we refer to $\mathrm{Ma}$. We use the Sweedler notation $\mathrm{Sw}$ whereby $\Delta a=a_{(1)} \otimes a_{(2)}$ and (id $\left.\otimes \Delta\right) \Delta a=a_{(1)} \otimes a_{(2)} \otimes a_{(3)}$, etc. We will frequently need the right adjoint coaction $\operatorname{Ad}_{R}(a)=a_{(2)} \otimes\left(S a_{(1)}\right) a_{(3)}$. We will write $A^{+} \subset A$ to denote the augmentation ideal (the kernel of $\epsilon$ ). We take $q \in \mathbb{C}$ invertible. General constructions work in fact over any field but our motivating point of view is over $\mathbb{C}$, which we retain for convenience.

We recall that a differential calculus of an algebra $A$ means an $A-A$-bimodule $\Omega^{1}$ and a map d : $A \rightarrow \Omega^{1}$ obeying the Leibniz rule and such that $\Omega^{1}$ is spanned by 1 -forms of the form $a \mathrm{~d} b$. A calculus on a Hopf algebra is left-covariant if the coproduct $\Delta: A \rightarrow A \otimes A$ viewed as a left coaction extends to a left coaction $\Delta_{L}$ on $\Omega^{1}$ such that $\mathrm{d}$ is an intertwiner and $\Delta_{L}$ is a bimodule map. In this case, having a Hopf-module, one knows that $\Omega^{1} \cong A \otimes \Lambda^{1}$ where $\Lambda^{1}$ are the left-invariant 1-forms, and that $\Lambda^{1}=A^{+} / I$ where $I$ is some right ideal contained in $A^{+}$Wo.

On the Hopf algebra $P=\mathbb{C}_{q}\left[S L_{2}\right]$ we take the 3 -d calculus of Wo. In our conventions this has a basis

$$
e^{-}=d \mathrm{~d} b-q b \mathrm{~d} d, \quad e^{+}=q^{-1} a \mathrm{~d} c-q^{-2} c \mathrm{~d} a, \quad e^{0}=d \mathrm{~d} a-q b \mathrm{~d} c
$$

left-invariant 1-forms, is spanned by these as a left module (according to the above) while the right module relations and exterior derivative are given in these terms by:

$$
e^{ \pm}\left(\begin{array}{ll}
a & b \\
c & d
\end{array}\right)=\left(\begin{array}{ll}
q a & q^{-1} b \\
q c & q^{-1} d
\end{array}\right) e^{ \pm}, \quad e^{0}\left(\begin{array}{ll}
a & b \\
c & d
\end{array}\right)=\left(\begin{array}{ll}
q^{2} a & q^{-2} b \\
q^{2} c & q^{-2} d
\end{array}\right) e^{0}
$$

$$
\mathrm{d} a=a e^{0}+q b e^{+}, \quad \mathrm{d} b=a e^{-}-q^{-2} b e^{0}, \quad \mathrm{~d} c=c e^{0}+q d e^{+}, \quad \mathrm{d} d=c e^{-}-q^{-2} d e^{0} .
$$

Our conventions for $e^{ \pm}$have been chosen with hindsight to fit the frame bundle geometry, see Theorem 3.1 The corresponding ideal is

$$
I_{P}=\left\langle a+q^{2} d-\left(1+q^{2}\right), b^{2}, c^{2}, b c,(a-1) b,(d-1) c\right\rangle .
$$

Next, we let $A=\mathbb{C}\left[t, t^{-1}\right]$ be a Hopf algebra with $\Delta t=t \otimes t$ and $S t=t^{-1}$. This coacts on $\mathbb{C}_{q}\left[S L_{2}\right]$, making it a comodule-algebra. Actually, a coaction here is the same thing a $\mathbb{Z}$-grading and in our case the degrees are

$$
\operatorname{deg}(a)=\operatorname{deg}(c)=1, \quad \operatorname{deg}(b)=\operatorname{deg}(d)=-1 .
$$

By definition the standard $q$-sphere $\mathbb{C}_{q}\left[S^{2}\right]$ is the degree zero (i.e. invariant) subalgebra of $\mathbb{C}_{q}\left[S L_{2}\right]$. It is a polynomial algebra $\mathbb{C}\left\langle b_{0}, b_{ \pm}\right\rangle$with inherited relations

$$
b_{ \pm} b_{0}=q^{ \pm 2} b_{0} b_{ \pm} \quad q^{2} b_{-} b_{+}=q^{-2} b_{+} b_{-}+\left(1-q^{-2}\right) b_{0}
$$

$$
b_{0}\left(1+q b_{0}\right)=b_{+} b_{-} .
$$

This last can also be written as $b_{0}\left(1+q^{-1} b_{0}\right)=q^{2} b_{-} b_{+}$. Here $b_{0}=b c, b_{+}=c d$ and $b_{-}=a b$. The first line of relations become as $q \rightarrow 1$ that the algebra is 
commutative, while (3) becomes the sphere relation in term of $b_{ \pm}$complex and $b_{0}+1 / 2$. Moreover, the coproduct of $\mathbb{C}_{q}\left[S L_{2}\right]$ restricts to $\mathbb{C}_{q}\left[S^{2}\right]$ as a left coaction $\Delta_{L}: \mathbb{C}_{q}\left[S^{2}\right] \rightarrow \mathbb{C}_{q}\left[S L_{2}\right] \otimes \mathbb{C}_{q}\left[S^{2}\right]$. General (2-parameter) 'quantum spheres' from the point of view of left comodule algebras were obtained in Po1].

On this $q$-sphere we inherit a differential calculus from the one above. It is not free over $\mathbb{C}_{q}\left[S^{2}\right]$ so we do not have a basis. But it is spanned by

$$
\mathrm{d} b_{+}=\mathrm{d}(c d)=d^{2} e^{+}+c^{2} e^{-}, \quad \mathrm{d} b_{-}=\mathrm{d}(a b)=b^{2} e^{+}+a^{2} e^{-}
$$

$$
\mathrm{d} b_{0}=\mathrm{d}(b c)=q b d e^{+}+q a c e^{-}
$$

using the Leibniz rule and the relations above. The inherited bimodule structure is far from trivial and will be recovered below by our own means. It is equivalent to formulae in $\left[\mathrm{Po2}\right.$. The calculus inherits a left coaction of $\mathbb{C}_{q}\left[S L_{2}\right]$ extending its coaction on $\mathbb{C}_{q}\left[S^{2}\right]$.

Finally, the calculi in both cases extend to entire exterior algebras. For $\mathbb{C}_{q}\left[S L_{2}\right]$ the natural extension compatible with the super-Leibniz rule on higher forms and $\mathrm{d}^{2}=0$ is:

$$
\begin{gathered}
\mathrm{d} e^{0}=q^{3} e^{+} \wedge e^{-}, \quad \mathrm{d} e^{ \pm}=\mp q^{ \pm 2}\left[2 ; q^{-2}\right] e^{ \pm} \wedge e^{0}, \quad\left(e^{ \pm}\right)^{2}=\left(e^{0}\right)^{2}=0 \\
q^{2} e^{+} \wedge e^{-}+e^{-} \wedge e^{+}=0, \quad e^{0} \wedge e^{ \pm}+q^{ \pm 4} e^{ \pm} \wedge e^{0}=0
\end{gathered}
$$

where $[n ; q]=\left(1-q^{n}\right) /(1-q)$ denotes a $q$-integer. This means that there are the same dimensions as classically, including a unique top form $e^{-} \wedge e^{+} \wedge e^{0}$. Again, these facts are well-known, but given here in our required conventions. For $\mathbb{C}_{q}\left[S^{2}\right]$ the exterior calculus is not so well-known and we obtain it below.

\section{Framings on NONUNiversal QUANTUM homogeneOUS SPACES}

The general formulation of a quantum principal bundle with nonuniversal calculi is as follows BM1, BM2. As 'total space coordinate ring' we have an algebra $P$. For the fiber a Hopf algebra $A$. We suppose that $P$ is a right $A$-comodule algebra by a coaction $\Delta_{R}$ and define the fixed subalgebra

$$
M=P^{A}=\left\{p \in P \mid \Delta_{R} p=p \otimes 1\right\}
$$

for the 'functions' on the base. For a bundle at the topological level we require that

$$
0 \rightarrow P\left(\Omega^{1} M\right) P \rightarrow \Omega^{1} P \stackrel{\text { ver }}{\longrightarrow} P \otimes A^{+} \rightarrow 0
$$

is exact where $\Omega^{1} P \subset P \otimes P$ is the universal calculus associated to any unital algebra (given by the kernel of the product map). The map on the right is $\operatorname{ver}\left(p \otimes p^{\prime}\right)=p \Delta_{R} p^{\prime}$, the generator of vertical vector fields. This exactness is equivalent to the similar map $P \otimes_{M} P \rightarrow P \otimes A$ being an isomorphism (a 'Hopf-Galois' extension $[\underline{S c h})$. When we have more general nonuniversal calculi then we require in addition that

$$
N_{M}=N_{P} \cap \Omega^{1} M
$$

$$
\Delta_{R} N_{P} \subseteq N_{P} \otimes A
$$

$$
\operatorname{ver}\left(N_{P}\right)=P \otimes I_{A}
$$


where $\Omega^{1}(P)=\left(\Omega^{1} P\right) / N_{P}$ etc defines the calculus on $P$ as the quotient of the universal one by a subbimodule. Here ([6) ensures that

$$
\Omega^{1}(M)=\operatorname{span}\left\{m \mathrm{~d}_{P} n \mid n, m \in M\right\} \subseteq \Omega^{1}(P)
$$

while (7) ensures that $\Omega^{1}(P)$ is left covariant. The coaction on $\Omega^{1} P$ here is the tensor product of the coaction on each $P$. Finally, (8) ensures that

$$
\text { ver : } \Omega^{1}(P) \rightarrow P \otimes \Lambda^{1}, \quad \Lambda^{1}=A^{+} / I_{A}
$$

is well-defined (the calculus on $A$ is assumed to be left-covariant as explained in the Preliminaries) and exactness of

$$
0 \rightarrow P \Omega^{1}(M) P \rightarrow \Omega^{1}(P) \stackrel{\text { ver }}{\longrightarrow} P \otimes \Lambda^{1} \rightarrow 0 .
$$

This is equivalent to the original formulation in BM1 based on such an exact sequence, as explained in BM2. In effect, we put differential structures and ensure that all relevant maps are compatible. Finally, in this theory, a connection is defined [BM1] as an equivariant splitting of $\Omega^{1}(P)$ providing a complement to the 'horizontal forms' $P \Omega^{1}(M) P$. If we assume that the calculus on $A$ is bicovariant then a connection is equivalent to an intertwiner

$$
\omega: \Lambda^{1} \rightarrow \Omega^{1}(P)
$$

such that ver $\circ \omega=1 \otimes \mathrm{id}$. Here $\Lambda^{1}$ has the right adjoint coaction inherited from that on $A^{+}$.

For the purposes of this paper, the main example is a 'quantum homogeneous bundle' BM1 BM2 based on a surjection $\pi: P \rightarrow A$ of Hopf algebras. This corresponds geometrically to an inclusion of groups, and just as the subgroup then acts by right multiplication, here $A$ coacts on $P$ by

$$
\Delta_{R}=(\text { id } \otimes \pi) \Delta: P \rightarrow P \otimes A .
$$

As above, we first construct the bundle with universal calculus and 'quantum homogeneous space base' $M=P^{A}$, and then impose differential structures. To do the latter we assume that $\Omega^{1}(P)$ is left-covariant and $\Omega^{1}(A)$ is bicovariant. We take (6) as a definition of $\Omega^{1}(M)$ while the remaining conditions (7)-(8) for a bundle with these nonuniversal calculi reduce to

$$
(\mathrm{id} \otimes \pi) \operatorname{Ad}_{R}\left(I_{P}\right) \subseteq I_{P} \otimes A, \quad \pi\left(I_{P}\right)=I_{A} .
$$

This follows immediately using $N_{P}=\left\{p S q_{(1)} \otimes q_{(2)} \mid p \in P, q \in I_{P}\right\}$ and computing $\Delta_{R}$, ver on such elements.

If one wants a connection, one can do this at the universal level via a bicovariant splitting map $i: A \rightarrow P$. Thus,

(11) $\Delta_{R} \circ i=(i \otimes \mathrm{id}) \Delta, \quad(\pi \otimes \mathrm{id}) \Delta \circ i=(\mathrm{id} \otimes i) \Delta \quad \Rightarrow \quad \omega(a)=\operatorname{Si}(a)_{(1)} \mathrm{d} i(a)_{(2)}$

is a connection. One in fact needs only a weaker $\mathrm{Ad}_{R}$-covariance condition [BM1] but the stronger bicovariance implies this $\mathrm{HM}$ and is the condition that is relevant below. In either case the map $i$ descends and defines a connection on the general bundle with nonuniversal calculus if

$$
i\left(I_{A}\right) \subseteq I_{P} .
$$

A further refinement of these constructions for quantum principal bundles can be found in BM2.

Up till now we have recalled the known quantum bundle construction itself. We are now ready to give the nonuniversal version of the frame bundle construction. 
An algebra $M$ is framed if it is the base of a quantum principal bundle as above to which $\Omega^{1}(M)$ is an associated bundle. The frame quantum group fiber need not be unique but its choice determines what kind of connections $\nabla$ on $\Omega^{1}(M)$ may be induced from connections on the frame bundle. More details are in M1, M2 . Apart from a bundle over $M$ as above, we need an $A$-comodule $V$. Then $\mathcal{E}=(P \otimes V)^{A}$ (the fixed submodule) plays the role of sections of the associated bundle. Finally, we need a 'soldering form' $\theta: V \rightarrow P \Omega^{1}(M)$ such that the induced left $M$-module map

is an isomorphism.

$$
s_{\theta}: \mathcal{E} \rightarrow \Omega^{1}(M), \quad p \otimes v \mapsto p \theta(v)
$$

Theorem 2.1. Let $\pi: P \rightarrow A$ be a quantum homogeneous bundle with general differential calculi as above. Then $M=P^{A}$ is framed by the bundle and

$$
\begin{gathered}
V=P^{+} \cap M / I_{P} \cap M, \quad \Delta_{R} v=\tilde{v}_{(2)} \otimes S \pi\left(\tilde{v}_{(1)}\right) \\
\theta(v)=S \tilde{v}_{(1)} \mathrm{d} \tilde{v}_{(2)}
\end{gathered}
$$

where $\tilde{v}$ is a representative of $v$ in $P^{+} \cap M$. Hence every quantum homogeneous space of this type is a 'quantum manifold' in the framed sense.

Proof. This construction for universal calculi is in [M1, Prop. 4.3] so we have mainly to check that various maps descend to the quotients needed for the nonuniversal calculi. First observe that $v \in M$ means by definition $v_{(1)} \otimes \pi\left(v_{(2)}\right)=v \otimes 1$. Moreover, if $v \in M$ then $v_{(1)} \otimes v_{(2)} \in P \otimes M$ because $v_{(1)} \otimes v_{(2)(1)} \otimes \pi\left(v_{(2)(2)}\right)=$ $v_{(1)(1)} \otimes v_{(1)(2)} \otimes \pi\left(v_{(2)}\right)=v_{(1)} \otimes v_{(2)} \otimes 1$. Similarly,

$$
\Delta_{R} v=v_{(1)} \otimes \pi\left(S v_{(1)}\right)=v_{(1)(2)} \otimes \pi\left(S v_{(1)(1)}\right) \pi\left(v_{(2)}\right)=v_{(2)} \otimes \pi\left(S v_{(1)} v_{(3)}\right)
$$

which is the projected adjoint action. Hence if $v \in I_{P} \cap M$ we see from (10) and from the above that $\Delta_{R} v \in I_{P} \cap M \otimes A$. Hence $\Delta_{R}$ descends to $V$. Incidentally, if $v \in P^{+} \cap M$ then $\epsilon\left(v_{(2)}\right) \pi\left(S v_{(1)}\right)=\pi(S v)=S \pi\left(v_{(2)}\right) \epsilon\left(v_{(1)}\right)=1 \epsilon(v)=0$ so $\Delta_{R}$ is defined on $P^{+} \cap M$ in the first place (this is the same as for the universal calculus case.) Meanwhile, if $v \in I_{P}$ then $S \tilde{v}_{(1)} \otimes \tilde{v}_{(2)} \in N_{P}$ and hence $\theta(v)=0$ in $\Omega^{1}(P)$, so this is well-defined. Moreover, if $\tilde{v} \in M$ is a representative of $v \in V$ then by the above remark, $\theta(v)=S \tilde{v}_{(1)} \mathrm{d} \tilde{v}_{(2)} \in P \Omega^{1}(M)$ as required. That $\theta$ is equivariant follows from this property proven for the universal calculi in M1 to which we refer for the proof. Hence all maps are defined as required and we have $s_{\theta}:(P \otimes V)^{A} \rightarrow \Omega^{1}(M)$. It remains to give its inverse, which we do by quotienting the inverse in the universal calculus case, namely

$$
s_{\theta}^{-1}(m \mathrm{~d} n)=\left[m n_{(1)} \otimes n_{(2)}-m n \otimes 1\right], \quad \forall m, n \in M
$$

where the expression in square brackets lies in $P \otimes P^{+} \cap M$ (again using the observation above) and [] denotes the equivalence class modulo $I_{P} \cap M$. That the result actually lies in $(P \otimes V)^{A}$ and gives the inverse of $s_{\theta}$ follows in the same way as in the universal case in M1.

Using $\omega$ such as from (11), one may define the covariant derivative

$$
D: \mathcal{E} \rightarrow \Omega^{1}(M) \underset{M}{\otimes} \mathcal{E}, \quad D=\left(\mathrm{id}-\Pi_{\omega}\right) \mathrm{d}
$$

where we apply $\mathrm{d} \otimes \mathrm{id}$ to $\mathcal{E}$ and $\Pi_{\omega}=\cdot(\mathrm{id} \otimes \omega)$ over is the vertical projection. When one takes the universal calculus this implies that $\mathcal{E}$ is a projective module and $D$ is the Grassmann connection associated to the projector [HM. In our case we get 
other connections which we will study in the next section. Also, using the framing, we obtain

$$
\nabla=\left(\mathrm{id} \otimes s_{\theta}^{-1}\right) \circ D \circ s_{\theta}: \Omega^{1}(M) \rightarrow \Omega^{1}(M) \underset{M}{\otimes} \Omega^{1}(M) .
$$

Both $D$ and $\nabla$ behave as covariant derivatives (so $\nabla(m \tau)=\mathrm{d} m \otimes_{M} \tau+m \nabla \tau$ for any function $m \in M$ and 1-form $\tau$ ). Hence we need only give $\nabla$ on exact forms.

Proposition 2.2. For the canonical connection induced by $i: A \rightarrow P$ on a quantum homogeneous space,

$$
\nabla(\mathrm{d} m)=\mathrm{d}\left(m_{(1)} S i \circ \pi\left(m_{(2)}\right)_{(1)}\right) \underset{M}{\otimes} i \circ \pi\left(m_{(2)}\right)_{(2)} S m_{(3)} \mathrm{d} m_{(4)} .
$$

This is the nonuniversal version of a similar formula with the universal differential calculus in M1. The proof is similar.

\section{Framing and holomorphic Calculus on standard $q$-SPhere $\mathbb{C}_{q}\left[S^{2}\right]$}

We start by recalling the known $q$-monopole bundle itself since we will need it in full detail when we use it as frame bundle. We fix the calculus on $P=\mathbb{C}_{q}\left[S L_{2}\right]$ to be the $3-\mathrm{d}$ one as explained in the Preliminaries. We take $A=\mathbb{C}\left[t, t^{-1}\right]$ and

$$
\pi(a)=t, \quad \pi(b)=\pi(c)=0, \quad \pi(d)=t^{-1} .
$$

The right coaction $\Delta_{R}=($ id $\otimes \pi) \Delta$ works out as

$$
\Delta_{R}\left(\begin{array}{ll}
a & b \\
c & d
\end{array}\right)=\left(\begin{array}{ll}
a & b \\
c & d
\end{array}\right) \otimes\left(\begin{array}{cc}
t & 0 \\
0 & t^{-1}
\end{array}\right)
$$

so that $\Delta_{R} a=a \otimes t$ etc. corresponding to $\operatorname{deg}(a)=1$, etc. Then $M=P^{A}=\mathbb{C}_{q}\left[S^{2}\right]$ as explained in the Preliminaries. It is known that we have a quantum homogeneous bundle with universal calculi. As in [BM1] we then take

$$
I_{A}=\pi\left(I_{P}\right)=\left\langle t+q^{2} t^{-1}-\left(1+q^{2}\right)\right\rangle=\left\langle(t-1)\left(t-q^{2}\right)\right\rangle
$$

(we factored $t^{-1}$ out of the generator obtained from projecting those of $I_{P}$ ). Now, this $I_{A}$ defines the 1 -dimensional calculus on $\mathbb{C}\left[t, t^{-1}\right]$ with basis $\mathrm{d} t=t \otimes[t-1]$ (where [ ] denotes modulo $I_{A}$ ) and relations

$$
\mathrm{d} t . t=t^{2} \otimes[t-1] t=t^{2} \otimes\left[(t-1)\left(t-q^{2}\right)\right]+t^{2} \otimes q^{2}[t-1]=q^{2} t^{2} \otimes[t-1]=q^{2} t \mathrm{~d} t
$$

This is a $q$-differential calculus whereby

$$
\mathrm{d}\left(t^{m}\right)=\left[m ; q^{2}\right] t^{m-1} \mathrm{~d} t
$$

so that the relevant partial derivative is the usual $q$-derivative.

We also verify that $I_{P}$ obeys the $\operatorname{Ad}_{R}$ condition in (10). Indeed, the element $a+q^{2} d$ (the $q$-trace) is $\operatorname{Ad}_{R}$-invariant. Meanwhile

$$
(\mathrm{id} \otimes \pi) \operatorname{Ad}_{R}\left(b^{2}\right)=b^{2} \otimes t^{-4}, \quad(\mathrm{id} \otimes \pi) \operatorname{Ad}_{R}\left(c^{2}\right)=c^{2} \otimes t^{4}
$$

and so forth. Hence we have the quantum sphere as a quantum homogeneous space where the calculus on it is obtained by restriction of that on $\mathbb{C}_{q}\left[S L_{2}\right]$ as required in (6).

Finally,

$$
i\left(t^{n}\right)=a^{n}, \quad i\left(t^{-n}\right)=d^{n}, \quad \forall n \geq 0
$$

defines a natural connection in the bundle via (11) as follows. We have

$$
i\left(I_{A}\right)=\operatorname{span}\left\{a^{m}(a-1)\left(a-q^{2}\right), a+q^{2} d-\left(1+q^{2}\right), d^{m}(d-1)\left(d-q^{2}\right)\right\} \subseteq I_{P} .
$$


The middle term is already in $I_{P}$. Hence also, multiplying it by $\left(a-q^{2}\right)$ we have

$$
(a-1)\left(a-q^{2}\right)+q^{2}(d-1)\left(a-q^{2}\right) \in I_{P} .
$$

The second term is $q^{2}\left(d a-1-q^{2} d-a+q^{2}+1\right)$ which lies in $I_{P}$ since $d a-1=$ $q b c \in I_{P}$. Hence $(a-1)\left(a-q^{2}\right) \in I_{P}$. Similarly for $(d-1)\left(d-q^{2}\right)$. Hence the canonical connection defined by this $i$ descends to the chosen nonuniversal calculi. The resulting $q$-monopole connection is

$$
\omega\left(t^{n}\right)=\left[n ; q^{2}\right] e^{0}
$$

for all integers $n$. This is easily proven by induction as follows. Thus, when $n \geq 0$,

$$
\begin{aligned}
& \omega\left(t^{n}\right)=\left(S a_{(1)}^{n} \mathrm{~d} a_{(2)}^{n}\right)=S\left(a_{(1)} a_{(1)}^{\prime} a_{(1)}^{\prime \prime} \cdots\right) \mathrm{d}\left(a_{(2)} a_{(2)}^{\prime} a_{(2)}^{\prime \prime} \cdots\right) \\
& =S\left(a_{(1)}^{\prime} a_{(2)}^{\prime \prime} \cdots\right) S a_{(1)}\left(\left(\mathrm{d} a_{(2)}\right) a_{(2)}^{\prime} a_{(2)}^{\prime \prime} \cdots+a_{(2)} \mathrm{d}\left(a_{(2)}^{\prime} a_{(2)}^{\prime \prime} \cdots\right)\right) \\
& =\omega\left(t^{n-1}\right)+S\left(a_{(1)}^{\prime} a_{(2)}^{\prime \prime} \cdots\right) \omega(t) a_{(2)}^{\prime} a_{(2)}^{\prime \prime} \cdots \\
& =\omega\left(t^{n-1}\right)+S\left(a_{(1)}^{\prime} a_{(2)}^{\prime \prime} \cdots\right) e^{0} a_{(2)}^{\prime} a_{(2)}{ }_{(2)} \cdots=\omega\left(t^{n-1}\right)+q^{2(n-1)} e^{0}
\end{aligned}
$$

where $a^{n}=a a^{\prime} a^{\prime \prime} \cdots$ is the product of $n$ copies of the generator $a \in \mathbb{C}_{q}\left[S L_{2}\right]$ (the primes are to keep the instances apart). We used the antimultiplicativity of the antipode $S$ and the Leibniz rules. We then used that $\omega(t)=\left(S a_{(1)}\right) \mathrm{d} a_{(2)}=$ $d \mathrm{~d} a-q b \mathrm{~d} c=e^{0}$ from the definition of the 3 - $\mathrm{d}$ calculus in the preliminaries. Finally, we used that $a_{(2)}^{\prime} a_{(2)}^{\prime \prime} \cdots$ has degree $n-1$ and hence its commutation relations with $e^{0}$ give a factor $q^{2(n-1}$ after which we cancel using the antipode axioms and $\epsilon(a)=1$. When we do the same with $n=-n^{\prime}, n^{\prime} \geq 0$ giving $\omega\left(t^{n}\right)=S d_{n_{(1)}} \mathrm{d} d_{{ }^{n^{\prime}}{ }_{(2)} \text { and a }}$ factor $q^{-2\left(n^{\prime}-1\right)}$ at the corresponding point. We also have $\omega\left(t^{-1}\right)=-q^{-2} e^{0}$. The two halves of the computation combine to the uniform answer (13). The curvature of the $q$-monopole connection is

$$
F_{\omega}\left(t^{n}\right)=\mathrm{d} \omega\left(t^{n}\right)+\omega\left(t^{n}\right) \wedge \omega\left(t^{n}\right)=\left[n, q^{2}\right] \mathrm{d} e^{0}=q^{3}\left[n ; q^{2}\right] e^{+} \wedge e^{-} .
$$

These constructions so far are not essentially new. They are a version of the $q$ monopole construction in BM1, BM2]. The choices and conventions are slightly closer to those in $[\mathrm{HM}]$ where, however, only universal calculi were considered.

Next, we compute $V$ in Theorem 2.1] Clearly $P^{+} \cap M=M^{+}=\operatorname{ker} \epsilon_{M}$. In our case $M=\mathbb{C}_{q}\left[S^{2}\right]$ is generated by $1, b_{ \pm}, b_{0}$ so that $M^{+}=\left\langle b_{0}, b_{ \pm}\right\rangle$as an ideal. Meanwhile, because $(a-1) b,(d-1) c, a+q^{2} d-\left(1+q^{2}\right)$ are not of homogeneous degree, the ideal which each one generates has no intersection with $M$. We assume here that $\mathbb{C}_{q}\left[S L_{2}\right]$ has no zero-divisors. We therefore focus on $b^{2}, c^{2}, b c$. The elements of degree zero in $\left\langle b^{2}\right\rangle$ include $b^{2}\left\{a^{2}, a c, c^{2}\right\}$. Hence, $b_{-}^{2}, b_{-} b_{0}, b_{0}^{2}$ lie in $I_{P} \cap M$. Similarly from $\left\langle c^{2}\right\rangle$ we have $b_{+}^{2}, b_{0} b_{+}$also in this ideal. The element $b c=b_{0}$ is already in the ideal. From these considerations we arrive at

$$
V=\left\langle b_{ \pm}\right\rangle /\left\langle b_{ \pm}^{2}, b_{0}\right\rangle .
$$

Hence $V$ is 2-dimensional with representatives $b_{ \pm}$. We then compute the coaction $\Delta_{R}$ on $V$ from Theorem 2.1] as

$$
\Delta_{R} b_{+}=c d \otimes S \pi\left(d^{2}\right)=b_{+} \otimes t^{2}, \quad \Delta_{R} b_{-}=a b \otimes S \pi\left(a^{2}\right)=b_{-} \otimes t^{-2} .
$$

Hence $V=\mathbb{C} \oplus \mathbb{C}$ and the associated bundle

$$
\mathcal{E}=\mathcal{E}_{-2} \oplus \mathcal{E}_{+2}=\mathbb{C}_{q}\left[S L_{2}\right]_{2} \oplus \mathbb{C}_{q}\left[S L_{2}\right]_{-2}
$$

is the direct sum of the $q$-monopole bundles of charge -2 and charge 2 . We identify their sections with the \pm 2 degree components in $\mathbb{C}_{q}\left[S L_{2}\right]$. Thus Theorem 2.1 yields: 
Theorem 3.1. $\mathbb{C}_{q}\left[S^{2}\right]=\mathbb{C}_{q}\left[S L_{2}\right]_{0}$ is a framed quantum manifold with cotangent bundle

$$
\Omega^{1}\left(\mathbb{C}_{q}\left[S^{2}\right]\right) \cong \mathcal{E}_{-2} \oplus \mathcal{E}_{2}
$$

isomorphic to the charge 2 and charge -2 monopole bundles. This isomorphism is given by the soldering form

$$
\begin{gathered}
\theta\left(b_{-}\right)=d^{2} \mathrm{~d} b_{-}+q^{2} b^{2} \mathrm{~d} b_{+}-\left[2 ; q^{2}\right] b d \mathrm{~d} b_{0}=e^{-} \\
\theta\left(b_{+}\right)=a^{2} \mathrm{~d} b_{+}+q^{-2} c^{2} \mathrm{~d} b_{-}-\left[2 ; q^{-2}\right] a c \mathrm{~d} b_{0}=e^{+}
\end{gathered}
$$

and makes $\Omega^{1}\left(\mathbb{C}_{q}\left[S^{2}\right]\right)$ projective.

Proof. The only remaining part is to compute $\theta\left(b_{ \pm}\right)$. We first find the coaction on $\mathbb{C}_{q}\left[S^{2}\right]$ inherited from the coproduct of $\mathbb{C}_{q}\left[S L_{2}\right]$ as

$$
\begin{gathered}
\Delta_{L}\left(b_{-}\right)=\Delta(a b)=a b \otimes\left(1+[2]_{q} b_{0}\right)+a^{2} \otimes b_{-}+b^{2} \otimes b_{+} \\
\Delta_{L}\left(b_{+}\right)=\Delta(c d)=c d \otimes\left(1+[2]_{q} b_{0}\right)+c^{2} \otimes b_{-}+d^{2} \otimes b_{+} \\
\Delta_{L}\left(b_{0}\right)=\Delta(b c)=1 \otimes b_{0}+b c \otimes\left(1+[2]_{q} b_{0}\right)+q a c \otimes b_{-}+q b d \otimes b_{+}
\end{gathered}
$$

where $[2]_{q}=q+q^{-1}$. These coproducts were already used in computing $\Delta_{R} b_{ \pm}$ above; this time we apply $S$ to the first factor and compute

$$
\theta\left(b_{+}\right)=S b_{+(1)} \mathrm{d} b_{+(2)}=-q^{-1} a c \mathrm{~d} b_{3}+a^{2} \mathrm{~d} b_{+}+q^{-2} c^{2} \mathrm{~d} b_{-}+q\left(-q^{-1}\right) a c \mathrm{~d} b_{3} q .
$$

Similarly for $\theta\left(b_{-}\right)$. This gives the middle expressions. We then insert (44) and find $e^{ \pm}$for the values of the map $\theta: V \rightarrow \Omega^{1}\left(\mathbb{C}_{q}\left[S L_{2}\right]\right)$. By Theorem 2.1 this is welldefined on $V$ and actually has its values in $\mathbb{C}_{q}\left[S L_{2}\right] \Omega^{1}\left(\mathbb{C}_{q}\left[S^{2}\right]\right)$. Also according to Theorem 2.1] one must multiply $\theta\left(b_{-}\right)$by an element of degree 2 , and $\theta\left(b_{+}\right)$by an element of degree -2 to get 1-forms on $\mathbb{C}_{q}\left[S^{2}\right]$ and every 1-form is obtained in this this way. $\mathcal{E}_{ \pm 2}$ are both projective as shown in [HM], as given via the Cuntz-Quillen theorem and the $q$-monopole connection with the universal calculus. $\diamond$

These two 1-forms $\theta\left(b_{ \pm}\right)=e^{ \pm}$play the role of 'vielbein' but do not themselves live on the base. Not also that as regards the bimodule structure, the elements of $\mathbb{C}_{q}\left[S^{2}\right]$ commute with the $\theta(v)$ as we see from (11).

Corollary 3.2. The three 1-forms $\mathrm{d} b_{ \pm}$and $\mathrm{d} b_{0}$ enjoy the relation

$$
q^{2} b_{-} \mathrm{d} b_{+}+b_{+} \mathrm{d} b_{-}-\left(1+[2]_{q} b_{0}\right) \mathrm{d} b_{0}=0
$$

where $[2]_{q}=q+q^{-1}$ denotes a symmetrized $q$-integer.

Proof. Using (16) we have $\theta\left(b_{0}\right)=S b_{0(1)} \mathrm{d} b_{0(2)}=b_{0} \mathrm{~d}\left(1+q b_{0}\right)+(-q) q b_{-} \mathrm{d} b_{+}+$ $\left(-q^{-1}\right) q b_{+} \mathrm{d} b_{-}+\left(1+q^{-1} b_{0}\right) \mathrm{d} b_{0}=0$ since $b_{0}$ represents zero in $V$. This identity can also be obtained from requiring $\mathrm{d} b_{ \pm}$and $\mathrm{d} b_{0}$ to be recovered from (4) composed with Theorem 3.1 and extensive use of the commutation relations.

This identity corresponds in the classical case to the differential of (3). However, for $q \neq 1$ this is not so immediate because the bimodule relations for $\Omega^{1}\left(\mathbb{C}_{q}\left[S^{2}\right]\right)$ are complicated to find explicitly. On the other hand, Theorem 3.1]implies a direct sum structure to the cotangent bundle with each piece more reasonable to work with. 
Corollary 3.3. $\Omega^{1}\left(\mathbb{C}_{q}\left[S^{2}\right]\right)=\Omega^{0,1} \oplus \Omega^{1,0}$ where $\Omega^{1,0}, \Omega^{0,1}$ are each first order leftcovariant differential calculi over $\mathbb{C}_{q}\left[S^{2}\right]$ with differentials $\partial, \bar{\partial}$ obeying $\mathrm{d}=\partial+\bar{\partial}$ and

$$
\begin{aligned}
& \partial b_{-}\left\{\begin{array}{l}
b_{+} \\
b_{-} \\
b_{0}
\end{array}=\left\{\begin{array} { l } 
{ q ^ { - 2 } b _ { + } \partial b _ { - } } \\
{ q ^ { 2 } b _ { - } \partial b _ { - } } \\
{ b _ { 0 } \partial b _ { - } , }
\end{array} \quad \partial b _ { + } \quad \left\{\begin{array}{l}
b_{+} \\
b_{-} \\
b_{0}
\end{array}=\left\{\begin{array}{l}
q^{2} b_{+} \partial b_{+} \\
q^{2} b_{-} \partial b_{+}+\left(q^{2}-q^{-2}\right) b_{+} \partial b_{-} \\
q^{4} b_{0} \partial b_{+}
\end{array}\right.\right.\right.\right. \\
& \bar{\partial} b_{-}\left\{\begin{array}{l}
b_{+} \\
b_{-} \\
b_{0}
\end{array}=\left\{\begin{array} { l } 
{ q ^ { - 2 } b _ { + } \overline { \partial } b _ { - } + ( q ^ { - 2 } - q ^ { 2 } ) b _ { - } \overline { \partial } b _ { + } } \\
{ q ^ { - 2 } b _ { - } \overline { \partial } b _ { - } } \\
{ q ^ { - 4 } b _ { 0 } \overline { \partial } b _ { - } , }
\end{array} \quad \overline { \partial } b _ { + } \left\{\begin{array}{l}
b_{+} \\
b_{-} \\
b_{0}
\end{array}=\left\{\begin{array}{l}
q^{-2} b_{+} \bar{\partial} b_{+} \\
q^{2} b_{-} \bar{\partial} b_{+} \\
b_{0} \bar{\partial} b_{+} .
\end{array}\right.\right.\right.\right.
\end{aligned}
$$

One has the relations

$$
\begin{aligned}
\partial b_{0} & =q^{2} b_{-} \partial b_{+}-q^{-2} b_{+} \partial b_{-}, & & \bar{\partial} b_{0}=b_{+} \bar{\partial} b_{-}-q^{4} b_{-} \bar{\partial} b_{+} \\
b_{0} b_{-} \partial b_{+} & =q^{-3}\left(1+q^{-1} b_{0}\right) b_{+} \partial b_{-}, & & b_{0} b_{+} \bar{\partial} b_{-}=q^{3}\left(1+q b_{0}\right) b_{-} \bar{\partial} b_{+} .
\end{aligned}
$$

Proof. From Theorem 3.1 know that $\Omega^{1}$ is a direct sum as a left module over $\mathbb{C}_{q}\left[S^{2}\right]$ spanned respectively by

$$
\left\{b^{2}, d b, d^{2}\right\} e^{+}=\left\{\partial b_{-}, \partial b_{0}, \partial b_{+}\right\}, \quad\left\{a^{2}, c a, c^{2}\right\} e^{-}=\left\{\bar{\partial} b_{-}, \bar{\partial} b_{0}, \bar{\partial} b_{+}\right\}
$$

where we use the expressions (41) to identify the two components of d. Next we observe that $e^{ \pm}$commute with elements of $\mathbb{C}_{q}\left[S^{2}\right]$ so that the commutation relations of functions with $\partial$ and $\bar{\partial}$ are easily determined from the relations of $\mathbb{C}_{q}\left[S L_{2}\right]$. We find the ones stated and

$\partial b_{0}\left\{\begin{array}{l}b_{+} \\ b_{-} \\ b_{0}\end{array}=\left\{\begin{array}{l}b_{+} \partial b_{0} \\ q^{4} b_{-} \partial b_{0}+q\left(1-q^{2}\right) \partial b_{-} \\ q^{2} b_{0} \partial b_{0},\end{array} \quad \bar{\partial} b_{0}\left\{\begin{array}{l}b_{+} \\ b_{-} \\ b_{0}\end{array}=\left\{\begin{array}{l}q^{-4} b_{+} \bar{\partial} b_{0}+q^{-1}\left(1-q^{-2}\right) \bar{\partial} b_{+} \\ b_{-} \bar{\partial} b_{0} \\ q^{-2} b_{0} \bar{\partial} b_{0} .\end{array}\right.\right.\right.\right.$

These close so that each $\partial, \bar{\partial}$ generate a bimodule differential calculus. Their Leibniz rules follow from that for $\mathrm{d}$ and the direct sum decomposition. Next, we observe the relations

$$
\begin{gathered}
b_{+} \partial b_{-}=q b_{0} \partial b_{0}, \quad b_{-} \partial b_{+}=q^{-2}\left(1+q^{-1} b_{0}\right) \partial b_{0} \\
b_{-} \bar{\partial} b_{+}=q^{-3} b_{0} \bar{\partial} b_{0}, \quad b_{+} \bar{\partial} b_{-}=\left(1+q b_{0}\right) \bar{\partial} b_{0}
\end{gathered}
$$

following likewise from the relations of $\mathbb{C}_{q}\left[S L_{2}\right]$ acting on $e^{ \pm}$. We use them as a definition of $\bar{\partial} b_{0}, \partial b_{0}$ and a relation among the $\bar{\partial} b_{ \pm}$(respectively, $\partial b_{ \pm}$) as stated. The latter also imply the relation in (3.2) and follow from differentiating (3), which is their geometrical content (related to the rank 1 projective module structure of each bundle). We note that one also has other relations, such as

$$
\begin{gathered}
b_{+} \partial b_{0}=q^{2} b_{0} \partial b_{+}, \quad b_{-} \partial b_{0}=q^{-1}\left(1+q^{-1} b_{0}\right) \partial b_{-} \\
b_{-} \bar{\partial} b_{0}=q^{-2} b_{0} \bar{\partial} b_{-}, \quad b_{+} \bar{\partial} b_{0}=q\left(1+q b_{0}\right) \bar{\partial} b_{+}
\end{gathered}
$$

which are not independent of the one already found. For example the $\bar{\partial}$ relations here can be written as

$$
b_{-}^{2} \bar{\partial} b_{+}=q^{-7} b_{0}^{2} \bar{\partial} b_{-}, \quad b_{+}^{2} \bar{\partial} b_{-}=q\left(1+q^{3} b_{0}\right)\left(1+q b_{0}\right) \bar{\partial} b_{+}
$$

which can be deduced from the one stated if one assumes that the left action of $b_{0}$ and $1+q^{-1} b_{0}$ respectively can be cancelled. Finally, each of the spaces $\Omega^{1,0}$ and $\Omega^{0,1}$ are stable under the left coaction of $\mathbb{C}_{q}\left[S L_{2}\right]$. This is because 'upstairs' on $\Omega^{1}\left(S L_{2}\right)$ the coaction on an element $f e^{ \pm}$is just $f_{(1)} \otimes f_{(2)} e^{ \pm}$and the coproduct 
defines a left coaction in each degree (because left-comultiplication commutes with the right-comultiplication used in defining the grading according to (id $\otimes \pi) \Delta$ ). This coaction is intertwined by $\partial$ and $\bar{\partial}$ with the left coaction (16) on $\mathbb{C}_{q}\left[S^{2}\right]$ since this is true for $\mathrm{d}$. $\diamond$

Clearly these 'holomorphic' and 'antiholomorphic' cotangent bundles are relatively simple to work with. On the other hand, we can use Theorem 3.1 to compute $\partial, \bar{\partial}$ in terms of d. Using the relations in $\mathbb{C}_{q}\left[S L_{2}\right]$ and Corollary 3.2$]$ we find

$$
\begin{gathered}
\partial b_{-}=q b_{-} \mathrm{d} b_{0}-q^{-1} b_{0} \mathrm{~d} b_{-}, \quad \partial b_{+}=\left(1+q b_{0}\right) \mathrm{d} b_{+}-q^{-1} b_{+} \mathrm{d} b_{0} \\
\partial b_{0}=q^{2} b_{-} \mathrm{d} b_{+}-q^{-1} b_{0} \mathrm{~d} b_{0}, \quad \bar{\partial} b_{0}=b_{+} \mathrm{d} b_{-}-q b_{0} \mathrm{~d} b_{0} \\
\bar{\partial} b_{-}=\left(1+q^{-1} b_{0}\right) \mathrm{d} b_{-}-q b_{-} \mathrm{d} b_{0}, \quad \bar{\partial} b_{+}=q^{-1} b_{+} \mathrm{d} b_{0}-q b_{0} \mathrm{~d} b_{+} .
\end{gathered}
$$

Therefore, as an application, we can recover the bimodule relations in $\Omega^{1}\left(\mathbb{C}_{q}\left[S^{2}\right]\right)$ from the much simpler ones for the two parts.

Proposition 3.4. Let $\mu=q^{2}-q^{-2}$. The bimodule relations for the 2-dimensional calculus on $\mathbb{C}_{q}\left[S^{2}\right]$ are

$$
\begin{gathered}
\mathrm{d} b_{0}\left\{\begin{array}{l}
b_{0} \\
b_{ \pm}
\end{array}=\left\{\begin{array}{l}
\left(q^{2}+q \mu b_{0}\right) b_{0} \mathrm{~d} b_{0}-\mu b_{0} b_{+} \mathrm{d} b_{-} \\
q^{\mp 2}\left(1 \mp q^{ \pm 1} \mu b_{0}\right) b_{ \pm} \mathrm{d} b_{0}-\left(1-q^{ \pm 2} \mp q^{ \pm 1} \mu b_{0}\right) b_{0} \mathrm{~d} b_{ \pm}
\end{array}\right.\right. \\
\mathrm{d} b_{ \pm}\left\{\begin{array}{l}
b_{0} \\
b_{\mp} \\
b_{ \pm}
\end{array}=\left\{\begin{array}{l}
\left(q^{ \pm 4} \pm q^{ \pm 1} \mu b_{0}\right) b_{0} \mathrm{~d} b_{+} \mp q^{\mp 1} \mu b_{ \pm} b_{0} \mathrm{~d} b_{0} \\
q^{ \pm 2}\left(1 \pm q^{ \pm 1} \mu b_{0}\right) b_{\mp} \mathrm{d} b_{ \pm} \pm q^{ \pm 1} \mu q^{-1} b_{0}^{2} \mathrm{~d} b_{0} \\
q^{ \pm 2}\left(1 \pm q^{ \pm 1} \mu b_{0}\right) b_{ \pm} \mathrm{d} b_{ \pm} \mp q^{\mp 1} \mu b_{ \pm}^{2} \mathrm{~d} b_{0} .
\end{array}\right.\right.
\end{gathered}
$$

Proof. These are all computed along the following lines:

$$
\mathrm{d} b_{0} \cdot b_{0}=q^{-2} b_{0} \bar{\partial} b_{0}+q^{2} b_{0} \partial b_{0}=q^{2} b_{0} \mathrm{~d} b_{0}+\left(q^{-2}-q^{2}\right) b_{0} \bar{\partial} b_{0}
$$

using $\mathrm{d}=\partial+\bar{\partial}$ and the commutation relations from Corollary 3.3. We then express $\bar{\partial} b_{0}$ in terms of $\mathrm{d}$ to obtain the result. Similarly for all the other commutation relations. $\diamond$

As a cross-check, one may now verify that Corollary 3.2 corresponds to the differentials of the the $q$-sphere relations. Put another way, using Corollary 3.2 the differentials of the four relations (3) of the $q$-sphere reduce to

$$
\begin{gathered}
q^{-1} \mathrm{~d} b_{+} \cdot b_{-}-\mathrm{d} b_{0} \cdot b_{0}=-q^{-2} b_{0} \mathrm{~d} b_{0}+q b_{-} \mathrm{d} b_{+} \\
q \mathrm{~d} b_{-} \cdot b_{+}-q^{-2} \mathrm{~d} b_{0} \cdot b_{0}=-b_{0} \mathrm{~d} b_{0}+q^{-1} b_{+} \mathrm{d} b_{-} \\
\mathrm{d} b_{ \pm} \cdot b_{0}-q^{ \pm 2} \mathrm{~d} b_{0} \cdot b_{ \pm}=q^{ \pm 2} b_{0} \mathrm{~d} b_{ \pm}-b_{ \pm} \mathrm{d} b_{0}
\end{gathered}
$$

which all hold using Proposition 3.4 In fact Podles in Po3 has shown that there is a unique left-covariant calculus on $\mathbb{C}_{q}\left[S^{2}\right]$ of the correct classical dimension, hence Proposition 3.4 is necessarily isomorphic to this, but derived differently. 


\section{Exterior algebra, Hodge-* And Maxwell theory on the $q$-SPhere}

In the last section we have expressed the cotangent bundle of $\mathbb{C}_{q}\left[S^{2}\right]$ as associated to a frame bundle by a $1 \oplus 1$-dimensional representation of the 'frame group' $\mathbb{C}\left[t, t^{-1}\right]$ equipped with a (bicovariant) $q$-differential structure. We deduced that $\Omega^{1}\left(\mathbb{C}_{q}\left[S^{2}\right]\right)$ is the sum of two 1-dimensional left-covariant calculi $\Omega^{0,1}$ and $\Omega^{1,0}$. We now extend this to the entire exterior algebra.

First of all, working 'upstairs' in $\Omega\left(\mathbb{C}_{q}\left[S L_{2}\right]\right)$ we define $\Omega\left(\mathbb{C}_{q}\left[S^{2}\right]\right)$ as the differential algebra obtained by restriction. It is generated by $\partial b_{ \pm}$and $\bar{\partial} b_{ \pm}$which means generated by $e^{ \pm}$and certain elements of $\mathbb{C}_{q}\left[S L_{2}\right]$. Because of the commutation relations with $e^{ \pm}$, these elements can all be collected to the left. Because of the relations between the $e^{ \pm}$, there is only a functional multiple of $e^{+} \wedge e^{-}$in degree 2 and nothing in higher degree, so

$$
\Omega^{2}\left(\mathbb{C}_{q}\left[S^{2}\right]\right)=\Omega^{1,1}, \quad \Omega^{2,0}=0=\Omega^{0,2}
$$

where the numbers refer to the degrees in $\partial, \bar{\partial}$ and $\Omega^{1,1}$ is 1 -dimensional over the algebra. We also extend $\partial$ and $\bar{\partial}$ by $\partial^{2}=\bar{\partial}^{2}=0$ so that each generates an exterior algebra (with top degree 1 by the above arguments 'upstairs' or from the relations in Corollary 3.3. Finally, we define

$$
\partial=\left.\mathrm{d}\right|_{\Omega^{0,1}}, \quad \bar{\partial}=\left.\mathrm{d}\right|_{\Omega^{1,0}}
$$

giving a double complex

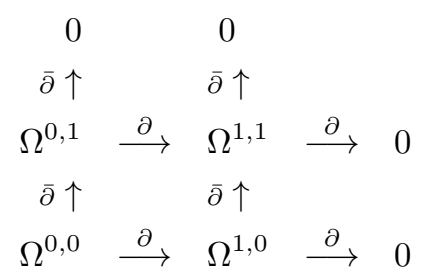

Here the graded-derivation property of $\mathrm{d}$ implies that

$$
\bar{\partial} \partial+\partial \bar{\partial}=0 .
$$

Moreover, forms which are left-invariant under the $\mathbb{C}_{q}\left[S L_{2}\right]$ coaction are precisely the ones generated by $e^{ \pm}$alone. Thus, up to scale, there is a unique left-invariant top form

$$
\Upsilon=e^{+} \wedge e^{-} \text {. }
$$

This is a basis of $\Omega^{2}\left(\mathbb{C}_{q}\left[S^{2}\right]\right)$ over $\mathbb{C}_{q}\left[S^{2}\right]$. We let $\mu=q^{2}-q^{-2}$.

Proposition 4.1. The relations between the $\Omega^{0,1}$ and $\Omega^{1,0}$ calculi are

$$
\begin{gathered}
\partial b_{+} \wedge \bar{\partial} b_{-}+q^{6} \bar{\partial} b_{-} \wedge \partial b_{+}=q^{4} \mu\left(b_{0}^{2}-1\right) \Upsilon \\
\partial b_{-} \wedge \bar{\partial} b_{+}=-q^{2} \bar{\partial} b_{+} \wedge \partial b_{-}=q^{2} b_{0}^{2} \Upsilon \\
\partial b_{-} \wedge \bar{\partial} b_{-}=-q^{6} \bar{\partial} b_{-} \wedge \partial b_{-}=q^{5} b_{-}^{2} \Upsilon \\
\partial b_{+} \wedge \bar{\partial} b_{+}=-q^{6} \bar{\partial} b_{+} \wedge \partial b_{+}=q^{5} b_{+}^{2} \Upsilon
\end{gathered}
$$

Proof. We compute all expressions in terms of $e^{ \pm}$using the definitions from the proof of Corollary 3.3] For example

$$
\bar{\partial} b_{-} \wedge \partial b_{+}=a^{2} e^{-} \wedge d^{2} e^{+}=q^{-2} a^{2} d^{2} e^{-} \wedge e^{+}=-\left(1+q^{-3} b_{0}\right)\left(1+q^{-1} b_{0}\right) \Upsilon
$$


using the relations between functions and $e^{ \pm}$in $\Omega^{1}\left(\mathbb{C}_{q}\left[S L_{2}\right]\right)$ and the relations in the quantum group. Computing all expressions in this way and comparing gives the relations stated. One may similarly compute

$$
\begin{array}{cc}
\partial b_{0} \wedge \bar{\partial} b_{0}=q^{4}\left(1+q b_{0}\right) b_{0} \Upsilon, & \bar{\partial} b_{0} \wedge \partial b_{0}=-\left(1+q^{-1} b_{0}\right) b_{0} \Upsilon \\
\partial b_{0} \wedge \bar{\partial} b_{-}=q^{4}\left(1+q b_{0}\right) b_{-} \Upsilon, & \bar{\partial} b_{-} \wedge \partial b_{0}=-\left(1+q^{-3} b_{0}\right) b_{-} \Upsilon \\
\partial b_{+} \wedge \bar{\partial} b_{0}=q^{4} b_{+}\left(1+q b_{0}\right) \Upsilon, & \bar{\partial} b_{0} \wedge \partial b_{+}=-b_{+}\left(1+q^{-3} b_{0}\right) \Upsilon \\
\partial b_{0} \wedge \bar{\partial} b_{+}=-q^{4} \bar{\partial} b_{+} \wedge \partial b_{0}=q^{3} b_{+} b_{0} \Upsilon, & \partial b_{-} \wedge \bar{\partial} b_{0}=-q^{4} \bar{\partial} b_{0} \wedge \partial b_{-}=q^{5} b_{-} b_{0} \Upsilon
\end{array}
$$

which will be useful later on, giving the further relations

$$
\begin{gathered}
q^{-4} \partial b_{0} \wedge \bar{\partial} b_{0}+\bar{\partial} b_{0} \wedge \partial b_{0}=\left(q-q^{-1}\right) b_{0}^{2} \Upsilon \\
\partial b_{0} \wedge \bar{\partial} b_{-}+q^{8} \bar{\partial} b_{-} \wedge \partial b_{0}=-q^{6} \mu b_{-} \Upsilon, \quad \partial b_{+} \wedge \bar{\partial} b_{0}+q^{8} \bar{\partial} b_{0} \wedge \partial b_{+}=-q^{6} \mu b_{+} \Upsilon . \diamond
\end{gathered}
$$

Note that first two lines taken together exhibit $\Upsilon$ as an element of $\Omega^{1,1}\left(\mathbb{C}_{q}\left[S^{2}\right]\right)$, which is otherwise not entirely clear (we will give another more geometrical expression later). One may further write it in terms of $d$ using results from the last section. For a simpler expression, the second line in Proposition 4.1 gives

$$
q b_{-} \mathrm{d} b_{0} \wedge \mathrm{d} b_{+}-q^{-1} b_{0} \mathrm{~d} b_{-} \wedge \mathrm{d} b_{+}=q^{2} b_{0}^{2} \Upsilon
$$

which gives the volume form if $b_{0}$ is invertible. An alternative is to use (4) and compute

$$
\mathrm{d} b_{+} \wedge \mathrm{d} b_{-}-q^{4} \mathrm{~d} b_{-} \wedge \mathrm{d} b_{+}=q^{3}[2]_{q}\left(1+[2]_{q} b_{0}\right) \Upsilon
$$

which gives the volume form if one assumes $1+[2]_{q} b_{0}$ invertible. These are classically the two trivialisations of the sphere given by deleting the north or south poles.

Next we look at the metric, motivated from Corollary 3.2 . We let $\bar{\otimes}$ denote the tensor product over $\mathbb{C}_{q}\left[S^{2}\right]$.

Proposition 4.2. There is a natural metric

$$
g=q^{2} \mathrm{~d} b_{-} \bar{\otimes} \mathrm{d} b_{+}+\mathrm{d} b_{+} \bar{\otimes} \mathrm{d} b_{-}-[2]_{q} \mathrm{~d} b_{0} \bar{\otimes} \mathrm{d} b_{0}
$$

such that $g$ is invariant under the left coaction of $\mathbb{C}_{q}\left[S L_{2}\right]$ and q-symmetric in the sense $\wedge(g)=0$. Moreover, $g \in\left(\Omega^{1,0} \bar{\otimes} \Omega^{0,1}\right) \oplus\left(\Omega^{0,1} \bar{\otimes} \Omega^{1,0}\right)$.

Proof. Left-invariance of the metric follows from

$$
\left(\begin{array}{ccc}
a^{2} & {[2]_{q} a b} & b^{2} \\
c a & 1+[2]_{q} b c & d b \\
c^{2} & {[2]_{q} c d} & d^{2}
\end{array}\right)^{t}\left(\begin{array}{ccc}
0 & 0 & q^{2} \\
0 & -[2]_{q} & 0 \\
1 & 0 & 0
\end{array}\right)\left(\begin{array}{ccc}
a^{2} & {[2]_{q} a b} & b^{2} \\
c a & 1+[2]_{q} b c & d b \\
c^{2} & {[2]_{q} c d} & d^{2}
\end{array}\right)=\left(\begin{array}{ccc}
0 & 0 & q^{2} \\
0 & -[2]_{q} & 0 \\
1 & 0 & 0
\end{array}\right)
$$

where $t$ denotes transpose and where we use the relations of $\mathbb{C}_{q}\left[S L_{2}\right]$. The transformation matrix here is the one in the coaction (16) in the basis $\mathrm{d} b_{-}, \mathrm{d} b_{0}, \mathrm{~d} b_{+}$. Actually, this coaction corresponds to the vector corepresentation of the even subalgebra $\mathbb{C}_{q}\left[S O_{3}\right]$ of $\mathbb{C}_{q}\left[S L_{2}\right]$ and for generic values of $q$ there is a unique invariant such matrix for the metric coefficients up to a scale. Hence the metric is uniquely determined if we suppose it has numerical coefficients with our basis of exact differentials are viewed as spanning a 3 -dimensional vector space over $\mathbb{C}$ (invariance at this level then implies invariance when viewed over $\bar{\otimes})$. Such numerical coefficients in turn are a natural assumption in view of (3.2). Differentiating that, we see that $\wedge(g)=0$. 
Also, writing $g=g_{++} \oplus g_{+-} \oplus g_{-+} \oplus g_{--}$for the decomposition according to Corollary 3.3. we use the $q$-commutation relations there and expressions for $\partial b_{i}$ in (17) to compute

$$
\begin{aligned}
g_{++}= & \partial b_{+} \bar{\otimes} \partial b_{-}-[2]_{q} \partial b_{0} \bar{\otimes} \partial b_{0}+q^{2} \partial b_{-} \bar{\otimes} \partial b_{+} \\
= & q^{3} b_{-} \partial b_{+} \bar{\otimes} \mathrm{d} b_{0}+\left(q^{3}-q^{-1}\right) b_{+} \partial b_{-} \bar{\otimes} \mathrm{d} b_{0}-q^{3} b_{0} \partial b_{+} \bar{\otimes} \mathrm{d} b_{-}+[2]_{q} b_{+} \partial b_{0} \bar{\otimes} \mathrm{d} b_{-} \\
& -[2]_{q}\left(1+q^{3} b_{0}\right) \partial b_{0} \bar{\otimes} \mathrm{d} b_{0}+q^{2}\left(1+q b_{0}\right) \partial b_{-} \bar{\otimes} \mathrm{d} b_{+}-q^{-1} b_{+} \partial b_{-} \bar{\otimes} \mathrm{d} b_{0} \\
= & -q^{-1}\left(1+q^{2}[2]_{q} b_{0}\right) \partial b_{0} \bar{\otimes} \mathrm{d} b_{0}+\left(q^{2}[2]_{q}-q^{3}\right) b_{0} \partial b_{+} \bar{\otimes} \mathrm{d} b_{-}+q^{2}\left(1+q b_{0}\right) \partial b_{-} \bar{\otimes} \mathrm{d} b_{+} \\
= & 0
\end{aligned}
$$

on using $\left(1+q^{2}[2]_{q} b_{0}\right) \partial b_{0}=\left(\partial b_{0}\right)\left(1+[2]_{q} b_{0}\right)$ and then using Corollary 3.2 to replace $\mathrm{d} b_{0}$ by $\mathrm{d} b_{ \pm}$. Then, as well as for the third equality, we used the relations in $\Omega^{1,0}$ in Corollary 3.3 to collect terms. There is a similar proof for $g_{--}=0$. $\diamond$

Next, we look at the Hodge $*$ operator

$$
*: \Omega^{1}\left(\mathbb{C}_{q}\left[S^{2}\right]\right) \rightarrow \Omega^{1}\left(\mathbb{C}_{q}\left[S^{2}\right]\right)
$$

which we require to obey $*^{2}=\mathrm{id}$ and to be at least a left-module map and to be frame-invariant (the metric can also be analysed in such terms but frame invariance alone does not fix a particular one, we used rotational left-covariance). In the frame bundle approach for $*$ we require $*: V \rightarrow V$ where $V$ is the 2-dimensional local tangent space. In order to be frame invariant (which means covariant under (15)) and square to the identity, this must be given by $*\left(e^{ \pm}\right)= \pm e^{ \pm}$up to an overall sign.

Proposition 4.3. The natural Hodge * operator is a left-covariant bimodule map obeying

$$
*(\partial f)=\partial f, \quad *(\bar{\partial} f)=-\bar{\partial} f, \quad \forall f \in \mathbb{C}_{q}\left[S^{2}\right] .
$$

and define a left-covariant lifting $i: \Omega^{2}\left(\mathbb{C}_{q}\left[S^{2}\right]\right) \rightarrow \Omega^{1}\left(\mathbb{C}_{q}\left[S^{2}\right]\right) \bar{\otimes} \Omega^{1}\left(\mathbb{C}_{q}\left[S^{2}\right]\right)$,

$$
i(\Upsilon)=\frac{q^{-1}}{[2]_{q}}(* \bar{\otimes} \mathrm{id})(g)=-\frac{q^{-1}}{[2]_{q}}(\mathrm{id} \bar{\otimes} *)(g)
$$

Proof. Let us first verify directly that $*$ is well-defined as a left module map, in which case it is given as stated since $\bar{\partial} b_{ \pm}, \bar{\partial} b_{0}$ are generated from $e^{-}$, etc. Indeed $\Omega^{1}\left(\mathbb{C}_{q}\left[S^{2}\right]\right)$ is a rank 2 bundle in which we can take $\mathrm{d} b_{ \pm}, \mathrm{d} b_{0}$ as generators with the relation in Corollary 3.2 . Writing $d=\partial+\bar{\partial}$ we have each part holding separately,

$$
q^{2} b_{-} \bar{\partial} b_{+}+b_{+} \bar{\partial} b_{-}-\left(1+[2]_{q} b_{0}\right) \bar{\partial} b_{0}=0
$$

and similarly for $\partial$ (this is also clear from relations in the proof of Corollary 3.3 ), so $*$ is compatible with this relation. That $*$ is a right $\mathbb{C}_{q}\left[S^{2}\right]$ bimodule map can easily be proven using the Leibniz rule for $\mathrm{d}, \bar{\partial}, \partial$. Moreover, $*$ is left-covariant under the coaction of $\mathbb{C}_{q}\left[S L_{2}\right]$ since the coaction acts on each $\Omega^{0,1}, \Omega^{1,0}$ separately. Next, we recall the usual formulae in which the Hodge $*$ operator is given in terms of the 'totally antisymmetric tensor' and the metric. The role of that tensor is played by the lifting of the volume form to an element of $\Omega^{1} \bar{\otimes} \Omega^{1}$ which is something that in classical geometry one takes for granted (the wedge product is given classically by skew-symmetrization). In noncommutative geometry, as explained in [M2], this 
lifting map is an additional datum required to split the wedge map $\Omega^{1} \bar{\otimes} \Omega^{1} \rightarrow \Omega^{2}$ and we use the Hodge ${ }^{*}$ operator and the metric to define it. We check that

$$
\begin{aligned}
\wedge(* \bar{\otimes} \mathrm{id})(g) & \\
= & q^{2}\left(-a^{2} e^{-}+b^{2} e^{+}\right) \wedge\left(c^{2} e^{-}+d^{2} e^{+}\right)+\left(-c^{2} e^{-}+d^{2} e^{+}\right) \wedge\left(a^{2} e^{-}+b^{2} e^{+}\right) \\
& -[2]_{q}\left(-c a e^{-}+d b e^{+}\right) \wedge\left(c a e^{-}+d b e^{+}\right) \\
= & \left(q^{2} a^{2} d^{2}+q^{4} b^{2} c^{2}+c^{2} b^{2}+q^{2} d^{2} a^{2}-[2]_{q} c a d b-q^{2}[2]_{q} d b c a\right) \Upsilon \\
= & \left(q^{2}+1\right) \Upsilon
\end{aligned}
$$

where we work 'upstairs' in the frame bundle and where the even terms give $q^{2}$ and the odd terms give 1 using the relations of $\mathbb{C}_{q}\left[S L_{2}\right]$. Hence $\wedge \circ i(\Upsilon)=\Upsilon$ as required. One may also obtain this result using the computations in the proof of Proposition 4.1 We define $i$ as by definition a left module map (it is not a bimodule map). The other stated expression for $i$ is the same in view of the form of $g$ in Proposition 4.2 Note also that since $\wedge(g)=0$ we have in fact a general family of lifts of this type,

$$
i(\Upsilon)=\frac{q^{-1}}{[2]_{q}}(* \bar{\otimes} \mathrm{id}) g+\mu g
$$

for any $\mu$. Or equivalently, $i(\Upsilon)=\alpha g_{+-}+\beta g_{-+}$provided $\alpha-\beta=2 q^{-1} /[2]_{q}$. These lifts are all left covariant under the coaction of $\mathbb{C}_{q}\left[S L_{2}\right]$ since $g_{+-}, g_{-+}$separately are. $\diamond$

We do not explicitly discuss complex structures in this paper; we work over $\mathbb{C}$ but with care this could be any field as in algebraic geometry. Nevertheless, our $b_{ \pm}$coordinates have their interpretation as complex linear combinations (of the ambient $\mathbb{R}^{3}$ coordinates) in real geometry; in real coordinates the Hodge $*$ is equivalent to an almost complex structure $J$ since this is defined in two dimensions exactly by the same relation between the volume form (viewed as a symplectic structure) and the metric as for $i$ in Proposition 4.3. In our case since we are deforming the standard metric on the sphere, this gives implicitly a $q$-deformation of its actual complex structure via the Hodge *. This justifies our notations $\partial, \bar{\partial}$.

We now have all the basic structures at least for the first 'layer' of geometry, namely cohomology and electromagnetism. For the Maxwell theory we define of course

$$
* 1=\Upsilon, \quad * \Upsilon=1
$$

and the Laplacian on degree zero by $\square=-\frac{1}{2} * \mathrm{~d} * \mathrm{~d}$. Then

$$
(\square f) \Upsilon=-\frac{1}{2} \mathrm{~d} * \mathrm{~d} f=\frac{1}{2} \mathrm{~d}(\bar{\partial} f-\partial f)=\partial \bar{\partial} f .
$$

Proposition 4.4. The functions $b_{-}, 1+[2]_{q} b_{0}, b_{+}$are eigenfunctions of $\square$ with eigenvalue $q^{2}[2]_{q}$.

Proof. We compute

$$
\mathrm{d} \partial\left\{\begin{array}{l}
b_{+} \\
b_{-} \\
b_{0}
\end{array} \quad=\left\{\begin{array}{l}
q \mathrm{~d} b_{0} \wedge \mathrm{d} b_{+}-q^{-1} \mathrm{~d} b_{+} \wedge \mathrm{d} b_{0} \\
q \mathrm{~d} b_{-} \wedge \mathrm{d} b_{0}-q^{-1} \mathrm{~d} b_{0} \wedge \mathrm{d} b_{-} \\
q^{2} \mathrm{~d} b_{-} \wedge \mathrm{d} b_{+}-q^{-1} \mathrm{~d} b_{0} \wedge \mathrm{d} b_{0}
\end{array}\right.\right.
$$


since $\mathrm{d}=\bar{\partial}$ on $\Omega^{1,0}$. We compute these using (4) in the same manner as in the proof of Proposition 4.3 Here we need their values explicitly:

$$
\begin{aligned}
\mathrm{d} b_{-} \wedge \mathrm{d} b_{+} & =-\left(1+q^{-2}[2]_{q} b_{0}-q^{-1}\left(q-q^{-1}\right)[3]_{q} b_{0}^{2}\right) \Upsilon \\
\mathrm{d} b_{0} \wedge \mathrm{d} b_{0} & =\left(q-q^{-1}\right) q^{2}\left([2]_{q}+[3]_{q} b_{0}\right) b_{0} \Upsilon \\
\mathrm{d} b_{0} \wedge \mathrm{d} b_{+} & =\left(\left(q^{5}-q^{-1}\right) b_{0}-1\right) b_{+} \Upsilon \\
\mathrm{d} b_{+} \wedge \mathrm{d} b_{0} & =\left(\left(q^{7}-q\right) b_{0}+q^{4}\right) b_{+} \Upsilon \\
\mathrm{d} b_{-} \wedge \mathrm{d} b_{0} & =b_{-}\left(\left(q^{5}-q^{-1}\right) b_{0}-1\right) \Upsilon \\
\mathrm{d} b_{0} \wedge \mathrm{d} b_{-} & =\left(q^{7}-q\right) b_{-} b_{0} \Upsilon
\end{aligned}
$$

which we use to find $\mathrm{d} \partial=\bar{\partial} \partial=-\partial \bar{\partial}$. $\quad \diamond$

Note that these functions form the vector corepresentation of $\mathbb{C}_{q}\left[\mathrm{SO}_{3}\right]$ under the left coaction (16) (this appears in the above basis as the transformation matrix in the proof of Proposition 4.2. In the same way, the matrix elements of each integer spin corepresentation $V_{n}$ of $\mathbb{C}_{q}\left[S L_{2}\right]$ define a square-dimension subspace $V_{n} \otimes V_{n}^{*} \subset \mathbb{C}_{q}\left[S L_{2}\right]$. Fixing the unique zero weight vector $v$ under the right coaction, the subspace $V \otimes v$ (in other words, the matrix entries in the middle row of the transformation matrix) span an eigenspace of the Laplacian. For generic $q$ the Peter-Weyl decomposition of $\mathbb{C}_{q}\left[S L_{2}\right]$ implies, as classically, that this is a complete diagonalisation of $\square$ on $\mathbb{C}_{q}\left[S^{2}\right]$ with one eigenspace for each integer spin corepresentation. The matrix elements of the $1 / 2$-integer spin corepresentations contain an odd number of the $\mathbb{C}_{q}\left[S L_{2}\right]$ generators and hence can never have the zero degree needed to lie in $\mathbb{C}_{q}\left[S^{2}\right]$.

In particular, the zero eigenspace of $\square$ is spanned by the constant function 1 . This implies that if $\bar{\partial} f=0$ then $f$ is a multiple of 1 . Similarly for $\partial$, so for the noncommutative de Rham and Dolbeault cohomology of $\mathbb{C}_{q}\left[S^{2}\right]$ for generic $q$ we have

$$
H^{0}=H_{\partial}^{0}=H_{\bar{\partial}}^{0}=\mathbb{C} .1 .
$$

It is clear that for generic $q$ we also have the usual values for the rest of the cohomology as well as Poincaré duality, since all constructions for this calculus are a smooth deformation of the classical ones. We omit explicit proofs of these facts since we need them for discussion only.

Similarly, we have a "massive" Maxwell equation defined on 1-forms by

$$
\square_{1} A \equiv-\frac{1}{4} * \mathrm{~d} * \mathrm{~d} A=m^{2} A, \quad * \mathrm{~d} * A=0
$$

The second equation is Coulomb gauge in physics and is automatic when $m \neq 0$ (in this case $m^{2} A$ should be interpreted as the source). We recall that in Maxwell theory the field is considered modulo exact forms but this freedom can be partially fixed by a gauge choice. We write the curvature as $F=\mathrm{d} A=f \Upsilon$ where $f=* F$ is in $\mathbb{C}_{q}\left[S^{2}\right]$. Then $\square_{1} A=0$ translates to

$$
\partial f=\bar{\partial} f=0
$$

which implies that $f \propto 1$. In that case, $\mathrm{d} A \propto \Upsilon$ which implies $f=0$ since $\Upsilon$ is not exact by Poincaré duality, so the only 'photons' are pure gauge. This is to be expected for a sphere. On the other hand, if $A$ is a "massive" mode, then

$$
A=-\frac{1}{m^{2}} * \mathrm{~d} f=\frac{1}{4 m}(\bar{\partial} f-\partial f), \quad \square f=2 m^{2} f .
$$


Conversely, given an eigenfunction of $\square$ as in the second equation, we use the first to define $A$ and obtain a massive mode. For example, the eigenfunctions of $\square$ in Proposition 4.4 give solutions

$$
A=\frac{q^{2}}{2[2]_{q}}\left(\bar{\partial} b_{i}-\partial b_{i}\right)
$$

where $i= \pm, 0$ and these are given as 1-forms via (17).

\section{Levi-Civita connection, Curvature and Dirac operator on the $q$-SPHERE}

Next, we compute use the frame bundle approach to develop the Riemannian geometry of the $q$-sphere. Here the $4 q$-monopole bundle [BM1] is viewed as the frame bundle and the $q$-monopole connection (13) on it as a spin connection. We find that it correctly induces the Levi-Civita connection on the cotangent bundle.

Theorem 5.1. The q-monopole connection (13) viewed as spin connection in the frame bundle of $\mathbb{C}_{q}\left[S^{2}\right]$ induces the covariant derivative

$$
\nabla\left\{\begin{array}{l}
\mathrm{d} b_{ \pm} \\
\mathrm{d} b_{0}
\end{array}=\left\{\begin{array}{c}
{[2]_{q} b_{ \pm}} \\
1+[2]_{q} b_{0}
\end{array}\right\} g\right.
$$

which is torsion-free and skew-metric compatible in the sense of zero cotorsion (a generalised Levi-Civita connection).

Proof. We recall that the bundle $\mathcal{E}_{-2}=\left(\mathbb{C}_{q}\left[S L_{2}\right] \otimes\left[b_{-}\right]\right)^{\mathbb{C}\left[t, t^{-1}\right]}$ can be identified with $\mathbb{C}_{q}\left[S L_{2}\right]_{2} \otimes\left[b_{-}\right]$, where we now write the representative $\left[b_{-}\right] \in V$ explicitly. This space in turn was identified with $\mathbb{C}_{q}\left[S L_{2}\right]_{2} \cdot e^{-}=\Omega^{0,1}$ as explained in the proof of Corollary 3.3. The action of the covariant derivative on $\mathcal{E}_{-2}$ is by

$D\left(f \otimes\left[b_{-}\right]\right)=\left(\mathrm{id}-\Pi_{\omega}\right)(\mathrm{d} f) \otimes\left[b_{-}\right]=\left(\mathrm{d} f-f \omega\left(t^{2}\right)\right) \otimes\left[b_{-}\right]=\left(\mathrm{d} f-\left(1+q^{2}\right) f e^{0}\right) \otimes\left[b_{-}\right]$ for all $f \in \mathbb{C}_{q}\left[S L_{2}\right]_{2}$. This is the usual covariant derivative on a $q$-monopole section, here of charge 2. Working 'upstairs' on $\mathbb{C}_{q}\left[S L_{2}\right]$ and using the Leibniz rule and the 3 -d calculus, we have

$$
\begin{gathered}
\mathrm{d}\left(a^{2}\right)=\left(1+q^{2}\right)\left(a^{2} e^{0}+q a b e^{+}\right), \quad \mathrm{d}(c a)=\left(1+q^{2}\right) c a e^{0}+q^{2}\left(1+[2]_{q} b c\right) e^{+} \\
\mathrm{d}\left(c^{2}\right)=\left(1+q^{2}\right)\left(c^{2} e^{0}+q c d e^{+}\right) .
\end{gathered}
$$

We see that the horizontal projection simply kills the $e^{0}$ term in each expression. Next, from the identity $d^{2} a^{2}+q^{2} b^{2} c^{2}-q[2]_{q} d b a c=1$ we write

$$
e^{+}=1 . e^{+}=q^{-2} \partial b_{+} . a^{2}+\partial b_{-} . c^{2}-q^{-2}[2]_{q} \partial b_{0} . c a
$$

where we move the $a, c$ generators to the right using the relations of the 3 -d calculus. These degree 2 products combine with $\left[b_{-}\right]$to give a section of $\mathcal{E}_{-2}$. We are working 'upstairs' but we can now identify the product as the $\mathbb{C}_{q}\left[S^{2}\right]$-module structure. Thus

$$
D\left(a^{2} \otimes\left[b_{-}\right]\right)=[2]_{q} b_{-}\left(\partial b_{+} .\left(a^{2} \otimes\left[b_{-}\right]\right)+q^{2} \partial b_{-} .\left(c^{2} \otimes\left[b_{-}\right]\right)-[2]_{q} \partial b_{0} \cdot\left(c a \otimes\left[b_{-}\right]\right)\right) .
$$

Similarly for $c a \otimes\left[b_{-}\right]$and $c^{2} \otimes\left[b_{-}\right]$. Finally, we replace $\left[b_{-}\right]$by $e^{-}$(the framing isomorphism Theorem 3.1) and identify the resulting elements of $\Omega^{0,1}$ on the right. This gives $\nabla \bar{\partial} b_{-}$. Similarly for all the other cases. For the $\nabla \partial$ we use

$$
e^{-}=q^{2} \bar{\partial} b_{-} . d^{2}+\bar{\partial} b_{+} \cdot b^{2}-[2]_{q} \bar{\partial} b_{0} . d b .
$$


As a result, we find

$$
\nabla\left(\partial b_{ \pm}\right)=[2]_{q} b_{ \pm} g_{-+}, \quad \nabla\left(\bar{\partial} b_{ \pm}\right)=[2]_{q} b_{ \pm} g_{+-}
$$

where

$$
g_{+-}=q^{2} \partial b_{-} \bar{\otimes} \bar{\partial} b_{+}+\partial b_{+} \bar{\otimes} \bar{\partial} b_{-}-[2]_{q} \partial b_{0} \bar{\otimes} \bar{\partial} b_{0}
$$

etc. in the decomposition of $g$ as in the proof of Proposition 4.2 Combining these gives $\nabla \mathrm{d}$ as stated.

Next, having found $\nabla$, we look at the torsion equation. As explained in M1 the noncommutative meaning of this is

$$
\text { Tor }=\nabla_{\wedge}-\mathrm{d}: \Omega^{1} \rightarrow \Omega^{2}
$$

which is the first degree measure of the failure of $\nabla \wedge$ to form a complex (the second degree measure is the curvature). We have

$$
\operatorname{Tor}\left(\mathrm{d} b_{ \pm}\right)=\nabla \wedge\left(\mathrm{d} b_{ \pm}\right)=[2]_{q} b_{ \pm} \wedge(g)=0
$$

by the $q$-symmetry in Proposition 4.2 Since the torsion is a left module map, it follows that the torsion vanishes entirely.

Finally, we look at the 'skew-metric compatibility' in the sense of zero cotorsion. This has been proposed as the correct notion of compatibility in M1 and can be written in terms of

$$
\text { CoTor }=(\nabla \wedge \text { id }- \text { id } \wedge \nabla) g \in \Omega^{2} \bar{\otimes} \Omega^{1}
$$

(there is an additional term if the torsion is not zero). Since the metric consists of exact differentials and since the torsion vanishes, the first $\nabla \wedge$ id vanishes. Looking at the second term, we compute

$$
\begin{aligned}
\frac{1}{[2]_{q}}(\mathrm{id} \wedge \nabla) g & =\frac{1}{[2]_{q}}\left(q^{2} \mathrm{~d} b_{-} \wedge \nabla \bar{\otimes} \mathrm{d} b_{+}+\mathrm{d} b_{+} \wedge \nabla \bar{\otimes} \mathrm{d} b_{-}\right)-\mathrm{d} b_{0} \wedge \nabla \bar{\otimes} \mathrm{d} b_{0} \\
& =q^{2} \mathrm{~d} b_{-} \wedge b_{+} g+\mathrm{d} b_{+} \wedge b_{-} g-\mathrm{d} b_{0} \wedge\left(1+[2]_{q} b_{0}\right) g \\
& =0
\end{aligned}
$$

by a right-module version $q^{2}\left(\mathrm{~d} b_{-}\right) b_{+}+\left(\mathrm{d} b_{+}\right) b_{-}-\mathrm{d} b_{0}\left(1+[2]_{q} b_{0}\right)=0$ of the relation in Corollary [3.2. Hence the cotorsion vanishes as well. $\diamond$

Note that the cotorsion or 'skew-metric compatibility' condition appropriate in noncommutative geometry M1 is weaker than the usual notion. In our case we have the more usual $\nabla g=O(q-1)$ (if $\nabla$ is taken to act on the tensor product as a derivation while keeping its left output to the far left), so that we recover the usual full metric compatibility only when $q=1$. It is also worth noting that viewed as sections of an associated bundle (see the Appendix), it is the $\bar{\partial} b_{ \pm}, \bar{\partial} b_{0}$ which are actually holomorphic in the sense $\nabla \partial b_{i} \in \Omega^{1,0} \bar{\otimes} \Omega^{1}$, rather than the image of $\partial$. Let us also use the connection to relate to the projective module point of view on quantum bundles.

Corollary 5.2. The projector

$$
E=\left(\begin{array}{c}
{[2]_{q} b_{-}} \\
1+[2]_{q} b_{0} \\
{[2]_{q} b_{+}}
\end{array}\right)\left(-b_{+}, 1+[2]_{q} b_{0},-q^{2} b_{-}\right)
$$

yields $\Omega^{1}\left(\mathbb{C}_{q}\left[S^{2}\right]\right)=\mathbb{C}_{q}\left[S^{2}\right]^{\oplus 3} .(1-E)$ and $\nabla=-E \mathrm{~d} E$ acting on $\mathrm{d} b_{-}, \mathrm{d} b_{0}, \mathrm{~d} b_{+}$. 
Proof. Note that proceeding from Theorem 3.1 would give a projector from 6 copies of $\mathbb{C}_{q}\left[S^{2}\right]$ whereas we provide a projector more in keeping with the classical geometrical picture from 3 copies. Moreover, we are not using the universal calculus as in $\mathrm{HM}$. Nevertheless, the form of $\nabla$ similarly suggests the projection shown, which we then verify directly. Thus, we have the dot products

$$
\left(-b_{+}, 1+[2]_{q} b_{0},-q^{2} b_{-}\right)\left(\begin{array}{c}
{[2]_{q} b_{-}} \\
1+[2]_{q} b_{0} \\
{[2]_{q} b_{+}}
\end{array}\right)=1, \quad\left(-b_{+}, 1+[2]_{q} b_{0},-q^{2} b_{-}\right)\left(\begin{array}{c}
\mathrm{d} b_{-} \\
\mathrm{d} b_{0} \\
\mathrm{~d} b_{+}
\end{array}\right)=0
$$

using respectively, the relations (3) of the $q$-sphere and the relation in Corollary 3.2 The second dot product with $\mathrm{d}$ of the row vector similarly gives $-g$. These observations imply that $E^{2}=E$ and (using the Leibniz rule to compute $\mathrm{d} E)$ that $\nabla=-\mathrm{d} E=-E \mathrm{~d} E$ when acting on the column vector $\left(\mathrm{d} b_{i}\right)$. Here $E$. $\left(\mathrm{d} b_{i}\right)=0$ (acting on the column vector). The map $\mathbb{C}_{q}\left[S^{2}\right]^{\oplus 3} \rightarrow \Omega^{1}\left(\mathbb{C}_{q}\left[S^{2}\right]\right)$ is given by $(f, g, h) \mapsto f \mathrm{~d} b_{-}+g \mathrm{~d} b_{0}+h \mathrm{~d} b_{+}=(f, g, h)(1-E)\left(\mathrm{d} b_{i}\right)$ and has kernel generated as a left module by the row vector in $E$. This corresponds to the relation in Corollary 3.2 Let us note that the same $E$ also gives the $\partial$ and $\bar{\partial}$ parts in a similar way. Thus $\nabla=-\partial E=-E \partial E$ when acting on the column vector $\left(\bar{\partial} b_{i}\right)$ and $\nabla=-\bar{\partial} E=-E \bar{\partial} E$ on $\left(\partial b_{i}\right)$. One may check that $\bar{\partial} E \cdot\left(\bar{\partial} b_{i}\right)=\partial E \cdot\left(\partial b_{i}\right)=0$ so that $\nabla=-\mathrm{d} E=-E \mathrm{~d} E$ when acting on either $\left(\partial b_{i}\right)$ or $\left(\bar{\partial} b_{i}\right)$ separately.

Proposition 5.3. The Riemann and Ricci tensors of the above generalised LeviCivita connection are

$$
\text { Riemann }\left.\right|_{\Omega^{0,1}}=[2]_{q} \Upsilon \bar{\otimes} \mathrm{id}, \quad \text { Riemann }\left.\right|_{\Omega^{1,0}}=-q^{4}[2]_{q} \Upsilon \bar{\otimes} \mathrm{id} .
$$

The lift

$$
i(\Upsilon)=\frac{q^{-1}}{[2]_{q}}\left(-(\operatorname{id} \bar{\otimes} *) g+\frac{1-q^{-4}}{1+q^{-4}} g\right)
$$

and trace in the middle position gives

$$
\text { Ricci }=\frac{2 q^{-1}}{1+q^{-4}} g
$$

making the q-sphere an 'Einstein space'.

Proof. The Riemann tensor is defined abstractly [M1 M2] by

$$
\text { Riemann }=(\text { id } \wedge \nabla-\mathrm{d} \bar{\otimes} \text { id }) \nabla: \Omega^{1} \rightarrow \Omega^{2} \bar{\otimes} \Omega^{1}
$$

as the form-version of the usual definition, as explained in M1. One may compute it from the formulae for $\nabla$ above. Since $\nabla\left(\bar{\partial} b_{ \pm}\right) \in \Omega^{1,0} \bar{\otimes} \bar{\partial}\left\{b_{i}\right\}$, when we apply id $\wedge \nabla$ we will get zero since $\Omega^{2,0}=0$. So only the $-\mathrm{d} \otimes$ id) $\nabla$ contributes. We have

$$
\begin{aligned}
-\frac{1}{[2]_{q}}(\mathrm{~d} \bar{\otimes} \mathrm{id}) \nabla\left(\bar{\partial} b_{+}\right)= & -b_{+}\left(q^{2} \bar{\partial} \partial b_{-} \bar{\otimes} \bar{\partial} b_{+}+\bar{\partial} \partial b_{+} \bar{\otimes} \bar{\partial} b_{-}-[2]_{q} \bar{\partial} \partial b_{0} \bar{\otimes} \bar{\partial} b_{0}\right) \\
& -\bar{\partial} b_{+} \wedge\left(q^{2} \partial b_{-} \bar{\otimes} \bar{\partial} b_{+}+\partial b_{+} \bar{\otimes} \bar{\partial} b_{-}-[2]_{q} \partial b_{0} \bar{\otimes} \bar{\partial} b_{0}\right) .
\end{aligned}
$$

We use Proposition 4.4 for the Laplacian $\bar{\partial} \partial$ and that $\Upsilon$ is central in the first line to collect $q^{2}[2]_{q} b_{+} \Upsilon$ to the left times (18), so that the first line vanishes. For the second line we use our computations for such wedge products in the proof of Proposition 4.1 as multiples of $\Upsilon$, to obtain

$$
-\frac{1}{[2]_{q}}(\mathrm{~d} \bar{\otimes} \mathrm{id}) \nabla\left(\bar{\partial} b_{+}\right)=\Upsilon\left(q^{2} b_{0}^{2} \bar{\otimes} \bar{\partial} b_{+}+q^{-1} b_{+}^{2} \bar{\otimes} \bar{\partial} b_{-}-[2]_{q} q^{-1} b_{+} b_{-} \bar{\otimes} \bar{\partial} b_{0}=\Upsilon \bar{\otimes} \bar{\partial} b_{+}\right.
$$


on using the relations of the $q$-sphere and the relations between the $\bar{\partial} b_{ \pm}, \bar{\partial} b_{0}$ in Corollary 3.3 Similarly for the Riemann tensor on $\bar{\partial} b_{-}$. The computation for $\operatorname{Riemann}\left(\partial_{ \pm}\right)$is similar but yields an extra factor $-q^{4}$ (the symmetry was broken in our choice of $\Upsilon$ ). We note that Riemann is a left module map so it is enough to find it on such exact differentials. It is also possible to compute the curvature 'upstairs' in the principal bundle, using (14). By the same conventions and explanations as in the proof of Theorem 5.1 we have, for example

$$
\operatorname{Riemann}\left(\partial b_{+}\right)=b^{2} F_{\omega}\left(t^{-2}\right) e^{+}=q^{3}\left[-2 ; q^{2}\right] b^{2} \Upsilon \bar{\otimes} e^{+}=-q^{4}[2]_{q} \Upsilon \bar{\otimes} \partial b_{+} .
$$

The curvature can be computed either way, as explained for the classical case in [M1. Using the Hodge $*$ operator we can write the Riemann tensor as

$$
\text { Riemann }=[2]_{q} \Upsilon \bar{\otimes}\left(\frac{1-q^{4}}{2}-\frac{1+q^{4}}{2} *\right) .
$$

For the Ricci tensor we need to lift the Riemann tensor to a map $\Omega^{1} \rightarrow \Omega^{1} \bar{\otimes} \Omega^{1} \bar{\otimes} \Omega^{1}$,

$$
\begin{aligned}
i(\text { Riemann }) & =[2]_{q} i(\Upsilon) \bar{\otimes}\left(\frac{1-q^{4}}{2}-\frac{1+q^{4}}{2} *\right) \\
& =\frac{2 q^{-1}}{1+q^{-4}}\left(\operatorname{id} \bar{\otimes}\left(\frac{1-q^{-4}}{2}-\frac{1+q^{-4}}{2} *\right)\right) g \bar{\otimes}\left(\frac{1-q^{4}}{2}-\frac{1+q^{4}}{2} *\right)
\end{aligned}
$$

where we use the lifting from the same family as in Proposition 4.3 but of the form stated. We can then take a trace by 'feeding' the right hand factor of $i(\Upsilon)$ into the input of Riemann. This gives

$$
\text { Ricci }=\frac{2 q^{-1}}{1+q^{-4}}\left(\operatorname{id} \bar{\otimes}\left(\frac{1-q^{4}}{2}-\frac{1+q^{4}}{2} *\right)\left(\frac{1-q^{-4}}{2}-\frac{1+q^{-4}}{2} *\right)\right) g
$$

which gives the result stated using $*^{2}=\mathrm{id} . \diamond$

The lift $i: \Omega^{2} \rightarrow \Omega^{1} \bar{\otimes} \Omega^{1}$ is an additional datum in the approach of M2 needed to define the Ricci tensor as well as interior products etc. Our point of view in the above Proposition [5.3 is that for the standard metric to be Einstein, the natural lift $i$ in Proposition 4.3 gets deformed by an additional $g$ component which vanishes as $q \rightarrow 1$. Equivalently, if we keep the choice of $i$ coming from the geometry in Proposition 4.3 then we find

$$
i(\Upsilon)=-\frac{q^{-1}}{[2]_{q}}(\mathrm{id} \bar{\otimes} *) g \quad \Rightarrow \quad \operatorname{Ricci}=\frac{q^{-1}\left(1+q^{4}\right)}{2} g+\frac{[2]_{q}\left(1-q^{4}\right)}{2} i(\Upsilon)
$$

which is a novel prediction of an 'antisymmetric' volume form correction to the Ricci tensor that vanishes as $q \rightarrow 1$.

Let us also note that the definition of Ricci used above is via the trace as in [M2], but between the second factor of the lift of Riemann (rather than the first factor as there) and its input. Also, we do not need a braided trace as was needed to keep covariance in the bicovariant calculus model in [M3], and do not have offsets $\theta \otimes \theta$ in Ricci as appeared there. The completely general definition of Ricci at the level of arbitrary framed algebras is not fully understood, but we see once again that in examples, as here, it is clear which trace to take.

The $\gamma$ matrices needed for the Dirac operator are likewise not yet formulated in the most general form for any framed algebra, but in examples there seems to be a clear choice. We propose the following. For the spin bundle we take

$$
\mathcal{S} \equiv \mathcal{S}_{-} \oplus \mathcal{S}_{+}=\mathcal{E}_{-1} \oplus \mathcal{E}_{+1}=\mathbb{C}_{q}\left[S L_{2}\right]_{1} \oplus \mathbb{C}_{q}\left[S L_{2}\right]_{-1}
$$


as given by the the monopole bundles of charges -1 and 1 . We identify the sections of the bundles with the degree \pm 1 subspaces of $\mathbb{C}_{q}\left[S L_{2}\right]$ as we did already for the charges \pm 2 . This corresponds to the double cover of the bundle for the cotangent space in Theorem 3.1 The next ingredient is a map

$$
\gamma: \Omega^{1}\left(\mathbb{C}_{q}\left[S^{2}\right]\right) \rightarrow \operatorname{End}(\mathcal{S})
$$

which we construct as follows. We use our description in Corollary 3.3 as we have throughout the paper to define

$$
\begin{aligned}
& \gamma: \Omega^{1,0} \bar{\otimes} \mathcal{S}_{-} \rightarrow \mathcal{S}_{+}, \quad \gamma\left(f e^{+} \bar{\otimes} \sigma\right)=f \sigma, \quad \forall f \in \mathbb{C}_{q}\left[S L_{2}\right]_{-2}, \quad \sigma \in \mathcal{S}_{-} \\
& \gamma: \Omega^{0,1} \bar{\otimes} \mathcal{S}_{+} \rightarrow \mathcal{S}_{-}, \quad \gamma\left(f e^{-\bar{\otimes} \tau}\right)=f \tau, \quad \forall f \in \mathbb{C}_{q}\left[S L_{2}\right]_{2}, \quad \tau \in \mathcal{S}_{+} .
\end{aligned}
$$

Here $\sigma, \tau$ denote appropriate sections and $\gamma$ under our identifications is nothing other than the product of $\mathbb{C}_{q}\left[S L_{2}\right]$ restricted to the appropriate degrees. We also let

$$
\left.\gamma\right|_{\Omega^{0,1} \bar{\otimes} \mathcal{S}_{-}}=0,\left.\quad \gamma\right|_{\Omega^{1,0} \bar{\otimes} \mathcal{S}_{+}}=0
$$

The classical motivation for $\gamma$ is as follows. Since $\partial$ is like a holomorphic differential one may think of it is a complex linear combination of the usual differentials. Likewise, if $\sigma^{1,2}$ are the usual Pauli matrices, then

$$
\frac{1}{2}\left(\sigma^{1}+\imath \sigma^{2}\right)=\left(\begin{array}{ll}
0 & 1 \\
0 & 0
\end{array}\right), \quad \frac{1}{2}\left(\sigma^{1}-\imath \sigma^{2}\right)=\left(\begin{array}{ll}
0 & 0 \\
1 & 0
\end{array}\right)
$$

which is the structure we have used for (24)-(26).

Lemma 5.4. The operator $\gamma: \Omega^{1}\left(\mathbb{C}_{q}\left[S^{2}\right]\right) \bar{\otimes} \mathcal{S} \rightarrow \mathcal{S}$ defined above is covariant under the left coaction of $\mathbb{C}_{q}\left[S L_{2}\right]$ and obeys

$$
\left\{\gamma_{\mathrm{d} b_{ \pm}}, \gamma_{* \mathrm{~d} b_{ \pm}}\right\}=0, \quad \gamma_{\mathrm{d} b_{ \pm}} \circ \gamma_{\mathrm{d} b_{ \pm}}=\left(\begin{array}{cc}
q^{-1} & 0 \\
0 & q^{3}
\end{array}\right) b_{ \pm}^{2}=-\gamma_{* \mathrm{~d} b_{ \pm}} \circ \gamma_{* \mathrm{~d} b_{ \pm}}
$$

where $\gamma_{\mathrm{d} b_{ \pm}}=\gamma\left(\mathrm{d} b_{ \pm} \bar{\otimes}()\right)$, etc. Moreover,

$$
\gamma \circ \gamma(g \bar{\otimes}())=\left(\begin{array}{ll}
q^{2} & 0 \\
0 & 1
\end{array}\right) \text { id }
$$

Proof. The left coaction on our various spaces is simply the coproduct $\Delta$ of $\mathbb{C}_{q}\left[S L_{2}\right]$ restricted to the appropriate degree. Since this is an algebra homomorphism, the $\gamma$ as given by the product is covariant. Likewise, $\partial b_{i}, \bar{\partial} b_{i}$ correspond as in Corollary 3.3 to $b^{2}, d b, d^{2}, a^{2}, c a, c^{2}$ and the relations among the corresponding $\gamma_{\partial b_{i}}, \gamma_{\bar{\partial} b_{i}}$ are just the relations among these generators of $\mathbb{C}_{q}\left[\mathrm{SO}_{3}\right]$ defined as the even part of $\mathbb{C}_{q}\left[S L_{2}\right]$, since $\gamma$ acts by left multiplication. Thus

$$
\begin{aligned}
& \gamma_{\partial b_{-}} \circ \gamma_{\bar{\partial} b_{-}}=b^{2} a^{2}=q^{3} b_{-}^{2}, \quad \gamma_{\bar{\partial} b_{-}} \circ \gamma_{\partial b_{-}}=q^{-1} b_{-}^{2} \\
& \gamma_{\partial b_{+}} \circ \gamma_{\bar{\partial} b_{+}}=d^{2} c^{2}=q^{3} b_{+}^{2}, \quad \gamma_{\bar{\partial} b_{+}} \circ \gamma_{\partial b_{+}}=q^{-1} b_{+}^{2} \\
& \gamma_{\partial b_{0}} \circ \gamma_{\bar{\partial} b_{0}}=d b c a=q^{2}\left(1+q b_{0}\right) b_{0}, \quad \gamma_{\bar{\partial} b_{0}} \circ \gamma_{\partial b_{0}}=\left(1+q^{-1} b_{0}\right) b_{0} \\
& \gamma_{\partial b_{+}} \circ \gamma_{\bar{\partial} b_{-}}=d^{2} a^{2}=\left(1+q^{3} b_{0}\right)\left(1+q b_{0}\right), \quad \gamma_{\bar{\partial} b_{-}} \circ \gamma_{\partial b_{+}}=\left(1+q^{-3} b_{0}\right)\left(1+q^{-1} b_{0}\right) \\
& \gamma_{\partial b_{-}} \circ \gamma_{\bar{\partial} b_{+}}=b^{2} c^{2}=b_{0}^{2}, \quad \gamma_{\bar{\partial} b_{+}} \circ \gamma_{\partial b_{-}}=b_{0}^{2} .
\end{aligned}
$$


The left column act on $\mathcal{S}_{+}$and the right column on $\mathcal{S}_{-}$by multiplication. Remembering that $\gamma$ acts by zero when the degrees do not match, we find $\gamma_{\mathrm{d} b_{ \pm}}=$ $\gamma_{\partial b_{ \pm}}+\gamma_{\bar{\partial} b_{ \pm}}$with square as stated. Since the Hodge $*$ changes the sign of $\bar{\partial} b_{i}$ we find similarly that $\gamma_{\mathrm{d} b_{ \pm}}$and $\gamma_{* \mathrm{~d} b_{ \pm}}$anticommute. Finally, the other expressions allow us to compute

$\gamma \circ \gamma\left(g_{+-} \bar{\otimes}()\right)=q^{2} b_{0}^{2}+\left(1+q^{3} b_{0}\right)\left(1+q b_{0}\right)-[2]_{q} q^{2}\left(1+q b_{0}\right) b_{0}=1, \quad \gamma \circ \gamma\left(g_{-+} \bar{\otimes}()\right)=q^{2}$ where for example $g_{+-}$is the $\Omega^{1,0} \bar{\otimes} \Omega^{0,1}$ component of the metric. These combine to the result stated.

The classical meaning of the $\gamma$ relations stated is that in a local coordinate chart the 2 -dimensional cotangent space is spanned by $\mathrm{d} b_{+}, * \mathrm{~d} b_{+}$, say, or (another chart) with $b_{-}$. We see that our $\gamma$ operators in these directions mutually anticommute and each square to a multiple of a $q$-deformation of the identity. The relation involving the metric is a weak form of the Clifford relations involving the metric, as proposed in $\mathrm{M} 2$.

Proposition 5.5. Let $D$ be the covariant derivative on $\mathcal{S}$ given by the $q$-monopole as spin connection. We define the Dirac operator on $\mathbb{C}_{q}\left[S^{2}\right]$ by

$$
\not \nabla=\gamma \circ D=\left(\begin{array}{cc}
0 & \gamma \circ \overline{\mathfrak{d}} \\
\gamma \circ \mathfrak{d} & 0
\end{array}\right): \mathcal{S} \rightarrow \mathcal{S}
$$

where $D=\mathfrak{d}+\overline{\mathfrak{d}}$ according to the parts in $\Omega^{1,0}$ and $\Omega^{0,1}$. Then $\not$ is covariant under the left coaction of $\mathbb{C}_{q}\left[S L_{2}\right]$ and under local frame rotations $\mathbb{C}\left[t, t^{-1}\right]$. Moreover, for $f=b_{-}, 1+[2]_{q} b_{0}, b_{+}$, we have

$$
\begin{aligned}
& \not^{2}(f a)=q^{-1}[2]_{q}(\square f) a+\left\{\begin{array}{l}
0 \\
-q^{-1} a \\
-q^{-1} c,
\end{array} \quad \nabla^{2}(f b)=q^{-1}[2]_{q}(\square f) b+\left\{\begin{array}{l}
0 \\
q b \\
q d
\end{array}\right.\right. \\
& \not^{2}(f c)=q^{-1}[2]_{q}(\square f) c+\left\{\begin{array}{l}
a \\
q c \\
0,
\end{array} \quad \nabla^{2}(f d)=q^{-1}[2]_{q}(\square f) d+\left\{\begin{array}{l}
-q^{2} b \\
-q^{3} d \\
0 .
\end{array}\right.\right.
\end{aligned}
$$

Proof. If $\sigma \in \mathcal{S}_{-}$then $\gamma(D \sigma)=\gamma(\mathfrak{d} \sigma) \in \mathcal{S}_{+}$etc., giving the stated form of $\not$ on $\mathcal{S}_{-} \oplus \mathcal{S}_{+}$. The space $\mathcal{S}_{-}$viewed as the degree 1 subspace of $\mathbb{C}_{q}\left[S L_{2}\right]$ is spanned over $\mathbb{C}_{q}\left[S^{2}\right]$ by $a, c$. These are not linearly independent but obey the relations

$$
b_{+} a-\left(1+q b_{0}\right) c=0, \quad b_{0} a-q^{2} b_{-} c=0
$$

as used in the projector $\mathrm{HM}$. The covariant derivative on such sections is known already from [BM1] and takes the form

$D a=\mathrm{d} a-a e^{0}=q b e^{+}=q^{-1} \partial b_{0} . a-q \partial b_{-} . c, \quad D c=\mathrm{d} c-c e^{0}=q d e^{+}=\partial b_{+} . a-q \partial b_{0} . c$

by similar computations as in Theorem 5.1 We omit writing the basis of the degree -1 left comodule $V=\mathbb{C}$ in view of our identifications. Then

$$
\not \nabla a=\gamma\left(b d e^{+} \bar{\otimes} a\right)-q \gamma\left(b^{2} e^{+} \bar{\otimes} c\right)=b d a-q b^{2} c=b .
$$

By such calculations, one has

$$
\not \nabla a=b, \quad \not c=d, \quad \not b=q a, \quad \not d d=q c
$$

where $b, d \in \mathcal{S}_{+}$and $a, c \in \mathcal{S}_{-}$. 
For $\not^{2}$ we note the Leibniz rule for any $\sigma \in \mathcal{S}_{-}$(say) and $f \in \mathbb{C}_{q}\left[S^{2}\right]$,

$$
\not \nabla(f \sigma)=f \not \nabla \sigma+\gamma(\partial f \bar{\otimes} \sigma)=f \not \nabla \sigma+f_{i} \gamma\left(\partial b_{i} \bar{\otimes} \sigma\right)
$$

where $\partial f=f_{i} \partial b_{i}$ (sum over $i=-, 0,+$ ) say. We similarly write $\bar{\partial} f=f_{i} \bar{\partial} b_{i}$ and have a similar expression for the Liebniz property on $\mathcal{S}_{+}$. We choose the coefficients $f_{i}$ from a fixed expansion $\mathrm{d} f=f_{i} \mathrm{~d} b_{i}$ (they are not unique). Then

$$
\not^{2}(f \sigma)=f \not^{2} \sigma+f_{i} \gamma\left(\bar{\partial} b_{i} \bar{\otimes} \not \nabla \sigma\right)+\gamma\left(\bar{\partial} f_{i} \bar{\otimes} \gamma\left(\partial b_{i} \bar{\otimes} \sigma\right)\right)+f_{i} \not \nabla \circ \gamma\left(\partial b_{i} \bar{\otimes} \sigma\right) .
$$

From Theorem 5.1 we have

$$
\nabla \partial f=\bar{\partial} f_{i} \bar{\otimes} \partial b_{i}+f_{i} \nabla \partial b_{i}=\bar{\partial} f_{i} \bar{\otimes} \partial b_{i}+f_{i}[2]_{q} b_{i} g_{-+}+f_{0} g_{-+}
$$

which combined with Lemma 5.4 gives the third term of $\nabla^{2}$ as

$$
\gamma \circ \gamma(\nabla \partial f \bar{\otimes} \sigma)-\left(q^{2} f_{i}[2]_{q} b_{i}+q^{2} f_{0}\right) \sigma .
$$

Meanwhile direct computation gives

$$
\not \nabla \circ \gamma\left(\partial b_{i} \bar{\otimes} \sigma\right)=\not\left(\left\{\begin{array}{ll}
b^{2} & \\
d b & . \sigma \\
d^{2} &
\end{array}\right)=\left(q^{2}[2]_{q} b_{i}+q^{2} \delta_{i, 0}\right) \sigma\right.
$$

at least when $\sigma=a, c$. Hence

$$
\not^{2}(f a)=q f a+f_{i} \gamma\left(\bar{\partial} b_{i} \bar{\otimes} b\right)+\gamma \circ \gamma(\nabla \partial f \bar{\otimes} a)
$$

and similarly for $\not^{2}(f c)$. Computing the middle terms, we find

$$
\begin{aligned}
& \nabla^{2}(f a)=q f a+q^{-1} f_{i} b_{i} a-q^{-1} f_{+} c+\gamma \circ \gamma(\nabla \partial f \bar{\otimes} a) \\
& \nabla^{2}(f c)=q f c+q^{-1} f_{i} b_{i} c+f_{-} a+f_{0} c+\gamma \circ \gamma(\nabla \partial f \bar{\otimes} c)
\end{aligned}
$$

for all $f \in \mathbb{C}_{q}\left[S^{2}\right]$. There are similar formulae for $\not^{2}(f b), \nabla^{2}(f d)$. The particular cases of $f$ stated then follow using Theorem 5.1 or (22) and $\gamma \circ \gamma(g \bar{\otimes}())$ from Lemma 5.4 We recognise the action of the Laplacian from Proposition 4.4 One may also obtain these particular cases by rather tedious direct computation, first finding

$$
\begin{aligned}
& \not \nabla\left(b_{i} a\right)=q[2]_{q} b_{i} b+\left\{\begin{array}{l}
0 \\
q b \\
d,
\end{array} \quad \not\left(b_{i} b\right)=[2]_{q} b_{i} a+\left\{\begin{array}{l}
0 \\
0 \\
-q^{-1} c
\end{array}\right.\right. \\
& \not \nabla\left(b_{i} c\right)=q[2]_{q} b_{i} d+\left\{\begin{array}{l}
-q b \\
0 \\
0,
\end{array} \quad \not\left(b_{i} d\right)=[2]_{q} b_{i} c+\left\{\begin{array}{l}
a \\
c \\
0 .
\end{array}\right.\right.
\end{aligned}
$$

where the cases are as $b_{i}=b_{-}, b_{0}, b_{+} \cdot \diamond$

The formulae for $\not^{2}$ are examples of 'Lichnerowicz type' formulae, where relative to a chosen (holomorphic or antiholomorphic) section, it is given by the scalar Laplacian from Proposition 4.4 plus an additional term, which we think of as some form of 'scaler curvature'. Let us also comment that our Dirac operator comes with a $\mathbb{Z}_{2}$ grading in the splitting $\mathcal{S}=\mathcal{S}_{-} \oplus \mathcal{S}_{+}$and $\not \nabla$ anticommutes with the grading. Also, from (28) and its cousin on $\mathcal{S}_{+}$, we have

$$
[\not \nabla, \hat{f}]=\gamma_{\mathrm{d} f}, \quad \forall f \in \mathbb{C}_{q}\left[S^{2}\right]
$$


where $\hat{f}$ denotes $f$ acting on $\mathcal{S}$ by left multiplication, so that $\not \nabla$ does allow one to recover the $\mathrm{d}$ of the calculus at the algebraic level. Thus we have some of the features of a spectral triple $\mathrm{Co}$ although not fitting precisely into that setting. On the other hand, our $\not$ naturally deforms the geometrical Dirac operator on the classical sphere and is motivated in that way rather than by such axioms. Also, it is not hard to exhibit some eigenfunctions of $\not 7$. From the proof of Proposition 5.5 we have

$$
\not\left(\begin{array}{c} 
\pm q^{\frac{1}{2}} a \\
b
\end{array}\right)= \pm q^{\frac{1}{2}}\left(\begin{array}{c} 
\pm q^{\frac{1}{2}} a \\
b
\end{array}\right), \quad \not\left(\begin{array}{c} 
\pm q^{\frac{1}{2}} c \\
d
\end{array}\right)= \pm q^{\frac{1}{2}}\left(\begin{array}{c} 
\pm q^{\frac{1}{2}} c \\
d
\end{array}\right)
$$

as solutions of the massive Dirac equation.

Finally, let us note that unlike the cotangent bundle, the spinor bundle is trivial. Both are trivial in $K$-theory having zero total monopole charge (see [HM]).

Proposition 5.6. Let $e=\left(\begin{array}{cc}-q^{-1} b_{0} & q b_{-} \\ -b_{+} & 1+q b_{0}\end{array}\right)$. Then

$$
\mathcal{S}_{-} \cong \mathbb{C}_{q}\left[S^{2}\right]^{\oplus 2}(1-e), \quad \mathcal{S}_{+} \cong \mathbb{C}_{q}\left[S^{2}\right]^{\oplus 2} e, \quad \mathcal{S} \cong \mathbb{C}_{q}\left[S^{2}\right]^{\oplus 2} .
$$

The covariant derivative and Dirac operators in the trivialisation are

$$
\begin{aligned}
& D=\mathrm{d}+(\mathrm{d} e) e-e \mathrm{~d} e . \\
& \not=\lambda q+\lambda q^{-1}\left(\overleftarrow{\partial}_{i} b_{i}\right)\left(1+\left(q^{4}-1\right) e\right) \\
& +\lambda q^{2}\left(\begin{array}{cc}
\overleftarrow{\partial}_{0} & q^{-1} \overleftarrow{\partial}_{+} \\
\overleftarrow{\partial}_{-} & 0
\end{array}\right) e-\lambda\left(\begin{array}{cc}
0 & q^{-1} \overleftarrow{\partial}_{+} \\
-\overleftarrow{\partial}_{-} & -\overleftarrow{\partial}_{0}
\end{array}\right)(1-e)
\end{aligned}
$$

acting on row 2-vectors, where $\mathrm{d} f=\left(f \overleftarrow{\partial}_{i}\right) \mathrm{d} b_{i}($ sum $i=-, 0,+)$ and $\lambda=q^{-\frac{1}{2}}$.

Proof. The projection for one half, the bundle $\mathcal{S}_{+}$, say, was obtained in [HM] with the universal calculus and this is our starting point. Our observation is that the projector for the other half of the spinor space is just given by the complementary projection. We then verify the desired properties directly. As spanning set for $\mathcal{S}_{-}, \mathcal{S}_{+}$we take the column vectors $\left(\begin{array}{l}a \\ c\end{array}\right)$ and $\left(\begin{array}{l}b \\ d\end{array}\right)$ respectively (these should not be confused with the $\mathcal{S}_{-} \oplus \mathcal{S}_{+}$column vectors above). We verify that

$$
\begin{gathered}
e\left(\begin{array}{l}
a \\
c
\end{array}\right)=0, \quad D\left(\begin{array}{l}
a \\
c
\end{array}\right)=-\partial e\left(\begin{array}{l}
a \\
c
\end{array}\right)=-e \mathrm{~d} e\left(\begin{array}{l}
a \\
c
\end{array}\right) \\
(1-e)\left(\begin{array}{l}
b \\
d
\end{array}\right)=0, \quad D\left(\begin{array}{l}
b \\
d
\end{array}\right)=\bar{\partial} e\left(\begin{array}{l}
b \\
d
\end{array}\right)=-(1-e) \mathrm{d}(1-e)\left(\begin{array}{l}
b \\
d
\end{array}\right)
\end{gathered}
$$

using the comutations above for $D$ and the relations (17) to find $\partial e=e$ de and $\bar{\partial} e=-(1-e) \mathrm{d}(1-e)=(\mathrm{d} e) e$.

The map $\mathbb{C}_{q}\left[S^{2}\right]^{\oplus 2} \rightarrow \mathcal{S}_{+}$is given by $(f, g) \mapsto f b+g d=(f, g) e\left(\begin{array}{l}b \\ d\end{array}\right)$. Simlarly for $\mathcal{S}_{-}$with $1-e$. Since given by complementary projectors, we see that together these maps trivialise $\mathcal{S}$. Let us write this explicitly as the combined map

$$
(f, g) \mapsto(f, g)\left(\begin{array}{c}
a+\lambda b \\
c+\lambda d
\end{array}\right)
$$


viewed in $\mathcal{S} \subset \mathbb{C}_{q}\left[S L_{2}\right]$. The $a, c$ have different degrees from $b, d$ so any nonzero value of $\lambda$ will do and yield an isomorphism. Noting that

$$
\partial e\left(\begin{array}{l}
b \\
d
\end{array}\right)=\bar{\partial} e\left(\begin{array}{l}
a \\
c
\end{array}\right)=0
$$

allows us to combine the expressions for $D$ according to

$$
D\left((f, g)\left(\begin{array}{l}
a+\lambda b \\
c+\lambda d
\end{array}\right)\right)=(\mathrm{d} f, \mathrm{~d} g) \bar{\otimes}\left(\begin{array}{l}
a+\lambda b \\
c+\lambda d
\end{array}\right)-(f, g)(e \mathrm{~d} e-(\mathrm{d} e) e)\left(\begin{array}{l}
a+\lambda b \\
c+\lambda d
\end{array}\right) .
$$

In view of the isomorphism, one can view this as an operator on $(f, g)$ which is as stated with $\mathrm{d}$ and matrix multiplication acting from the right (this is inevitable in our conventions).

For the Dirac operator we use the Leibniz formula (28) and set $\lambda=q^{-\frac{1}{2}}$ so that $a+\lambda b, c+\lambda d$ are eigenvectors of $\not \nabla$. Then

$$
\not\left((f, g)\left(\begin{array}{l}
a+\lambda b \\
c+\lambda d
\end{array}\right)\right)=\gamma_{(\mathrm{d} f, \mathrm{~d} g)}\left(\left(\begin{array}{l}
a+\lambda b \\
c+\lambda d
\end{array}\right)\right)+\lambda^{-1}(f, g)\left(\begin{array}{l}
a+\lambda b \\
c+\lambda d
\end{array}\right) .
$$

By similar methods as in the proof of Proposition [5.5 we write $\mathrm{d} f=f_{i} \mathrm{~d} b_{i}$ and take these coefficients also for expansions of $\partial f, \bar{\partial} f$. Then

$\gamma_{(\mathrm{d} f, \mathrm{~d} g)}\left(\left(\begin{array}{l}a+\lambda b \\ c+\lambda d\end{array}\right)\right)=\left(f_{i}, g_{i}\right) b_{i}\left(\begin{array}{c}q^{2} b+\lambda q^{-1} a \\ q^{2} d+\lambda q^{-1} c\end{array}\right)+\left(q f_{0}-q g_{-}, f_{+}\right)\left(\begin{array}{l}b \\ d\end{array}\right)+\lambda\left(g_{-},-q^{-1} f_{+}+g_{0}\right)\left(\begin{array}{l}a \\ c\end{array}\right)$.

By inserting an $e$ in front of $\left(\begin{array}{l}b \\ d\end{array}\right)$ and $(1-e)$ in front of $\left(\begin{array}{l}a \\ c\end{array}\right)$, we can write this as a certain operation on $(f, g)$ followed by dot product with $\left(\begin{array}{l}a+\lambda b \\ c+\lambda d\end{array}\right)$, which gives the $\not \nabla$ stated. Here $\overleftarrow{\partial}_{i}$ denotes the right-acting operator which assigns our chosen coefficients $f_{i}$ to $f$ and $g_{i}$ to $g$. The last two terms of $\nabla$ (the displayed matrix terms) can be combined as

$$
\lambda\left(\begin{array}{cc}
-q \overleftarrow{\partial}_{0} b_{0}-[2]_{q} \overleftarrow{\partial}_{+} b_{+} & q^{3} \overleftarrow{\partial}_{0} b_{-}+q \overleftarrow{\partial}_{+}\left(1+\overleftarrow{L}_{\partial_{0}}\right]_{q} b_{0} \\
\overleftarrow{\partial}_{0} b_{+}+\overleftarrow{\partial}_{-}\left(1+[2]_{q} b_{0}\right) & -q^{2}[2]_{q} \overleftarrow{\partial}_{-} b_{-}-q \overleftarrow{\partial}_{0} b_{0}
\end{array}\right)
$$

This part alone is not well-defined (recall that the chosen coefficients of $\mathrm{d} f, \mathrm{~d} g$ are not unique) but the entire $\not \nabla$ is well-defined and independent of the choice, as it must be since $\not \nabla$ exists geometrically on the bundle $\mathcal{S}$. $\diamond$

It remains to make some remarks about the classical limits. Setting $q=1$ we have

$$
\nabla_{q=1}=1+\left(\begin{array}{cc}
\overleftarrow{\partial}_{-} b_{-}-\overleftarrow{\partial}_{+} b_{+} & \overleftarrow{\partial}_{0} b_{-}+\overleftarrow{\partial}_{+}\left(1+2 b_{0}\right) \\
\overleftarrow{\partial}_{0} b_{+}+\overleftarrow{\partial}_{-}\left(1+2 b_{0}\right) & \overleftarrow{⿱}_{+} b_{+}-\overleftarrow{\partial}_{-} b_{-}
\end{array}\right)=1-\imath \sigma \cdot \overleftarrow{\partial} \times \overleftarrow{x}
$$

when we shift to usual $x, y, z$ coordinates related (say) as $b_{ \pm}= \pm(x \pm \imath y)$ and $b_{0}=z-\frac{1}{2}$, corresponding to a sphere of radius $1 / 2$ embedded in $\mathbb{R}^{3}$. Changing variables to $\overleftarrow{\partial}=\left(\overleftarrow{\partial}_{x}, \overleftarrow{\partial}_{y}, \overleftarrow{\partial}_{z}\right)$ (so that $\overleftarrow{\partial}_{ \pm}= \pm \frac{1}{2}\left(\overleftarrow{\partial}_{x} \mp \imath \overleftarrow{\partial}_{y}\right)$ and $\left.\overleftarrow{\partial}_{0}=\overleftarrow{\partial}_{z}\right)$ gives $\not$ as stated in terms of the usual Pauli matrices and the vector $\overleftarrow{x}=(x, y, z)$ acting by right multiplication. This expression makes sense on functions on $\mathbb{R}^{3}$ but vanishes 
on functions that depend only on the radius, and hence descends to functions on $S^{2}$. Incidentally, as is well-known, $\not^{2}=\not \nabla+\square$ where

$$
\square=(\overleftarrow{\partial} \cdot \overleftarrow{x})^{2}+\overleftarrow{\partial} \cdot \overleftarrow{x}-(\overleftarrow{\partial} \cdot \overleftarrow{\partial})(\overleftarrow{x} \cdot \overleftarrow{x})
$$

(an easy computation) and one may check that $\square$ defined on the ambient $\mathbb{R}^{3}$ again descends to $S^{2}$ and is indeed the classical limit of the Laplace operator in Proposition 4.4 under the same change of classical coordinates and $\overleftarrow{x} \cdot \overleftarrow{x}=1 / 4$. Obviously all operations here may be reformulated acting from the left as more usual.

\section{Appendix A. Geometrical $q$-Borel-Weil-Bott construction}

The Borel-Weil-Bott construction in classical representation theory constructs irreducible representations of a compact Lie group $G$ as follows. From the bundle $G \rightarrow G / T$ (where $T$ is the maximal torus) and an irreducible representation $V$ of $T$ (given by a character) we construct the associated bundle $E=G \times_{T} V$ over $G / T$. Its space of sections $\mathcal{E}$ still carries a representation of $G$ acting on $G / T$ from the left and lifted to an action on $\mathcal{E}$ by a certain connection. This space is, however, much too big to be an irreducible representation. From the point of view of 'geometric quantization' one must choose a polarization. A natural approach here is to use the complex structure on $G / T$ and take the holomorphic sections $\mathcal{E}^{\text {hol }}$. These are a much smaller space and form an irreducible representation of $G$. We refer to GZ for an excellent account of the classical situation from this point of view and of the quantum case from a representation theoretic (but not really geometric) point of view. On the other hand, in the course of understanding the geometry of the $q$ sphere, we have now obtained all the ingredients for the quantum group geometrical version of this construction. We outline this application in this appendix. The full details and generalisation from $\mathbb{C}_{q}\left[S L_{2}\right]$ to other quantum groups will be addressed elsewhere.

Indeed, this remark is about the monopole associated bundles and their covariant derivatives as we have already used for charges $\pm 2, \pm 1$. Now we consider general $\mathcal{E}_{-n}=\left(\mathbb{C}_{q}\left[S L_{2}\right] \otimes V\right)^{\mathbb{C}\left[t, t^{-1}\right]}$ where $V=\mathbb{C} \cdot v$ is the right comodule defined by $\Delta_{R} v=$ $v \otimes t^{-n}$. This is 1 -dimensional so that $\mathcal{E}_{n}=\mathbb{C}_{q}\left[S L_{2}\right]_{n} . v$, i.e. isomorphic to the degree $n$ component. As such $\mathcal{E}_{-n}$ also carries the left coaction of $\mathbb{C}_{q}\left[S L_{2}\right]$ given by restricting the coproduct since this respects the degree (the coaction on degree 0 was already used in (16)). Let $D$ be the usual covariant derivative for the monopole connection. We say that a section $\sigma \in \mathcal{E}_{-n}$ is holomorphic if

$$
D \sigma \in \Omega^{1,0} \bar{\otimes} \mathcal{E}_{-n} .
$$

In other words, if we write $D=\mathfrak{d}+\overline{\mathfrak{d}}$ for the $\Omega^{1,0}$ and $\Omega^{0,1}$ parts then we require $\overline{\mathfrak{d}} \sigma=0$.

Proposition A.1. The space of holomorphic sections $\mathcal{E}_{-n}^{\text {hol }}$ of the charge $-n q$ monopole bundle contains the standard $n+1$-dimensional corepresentation of $\mathbb{C}_{q}\left[S L_{2}\right]$.

Proof. Here $n \geq 0$. The standard $n+1$-dimensional corepresentation of $\mathbb{C}_{q}\left[S L_{2}\right]$ corresponds to the $q$-deformation of the standard $n+1$-dimensional irreducible representation of $S L_{2}$ and is given by

$$
\left\{c^{s} a^{t} \mid s+t=n, s, t \geq 0\right\} .
$$


These are all in the degree $n$ component of $\mathbb{C}_{q}\left[S L_{2}\right]$ and form a left corepresentation via the restriction of the coproduct. We verify that as such, they are homomorphic. Indeed, as in the computation for Theorem [5.1] we compute

$$
\mathrm{d} c^{s}=\left[s ; q^{2}\right] c^{s-1}\left(c e^{0}+q d e^{+}\right), \quad \mathrm{d} a^{t}=\left[t ; q^{2}\right] a^{t-1}\left(a e^{0}+q b e^{+}\right)
$$

as easily proven by induction. Then

$$
\mathrm{d}\left(c^{s} a^{t}\right)=\left[n ; q^{2}\right] c^{s} a^{t} e^{0}+q^{t} c^{s-1} a^{t-1}\left(q\left[s ; q^{2}\right]+\left[n ; q^{2}\right] b c\right) e^{+}
$$

by the Leibniz rule. Hence using (13) we have

$$
D\left(c^{s} a^{t}\right)=\mathrm{d}\left(c^{s} a^{t}\right)-c^{s} a^{t} \omega\left(t^{n}\right)=q^{t} c^{s-1} a^{t-1}\left(q\left[s ; q^{2}\right]+\left[n ; q^{2}\right] b c\right) e^{+} .
$$

The expressions are slightly simpler when $s=0$ or $t=0$. Next, we move $c^{s-1} a^{t-1}$ to the far right and use (21) to see that $D\left(c^{s} a^{t}\right) \in \Omega^{1,0} \bar{\otimes} \mathcal{E}_{-n}$ by the same argument as in the proof of Theorem 5.1 Hence these elements are holomorphic as claimed. $\diamond$

Conversely, if $f \in \mathbb{C}_{q}\left[S^{2}\right]$ and $\sigma \in \mathcal{E}_{-n}^{\text {hol }}$ then $D(f \sigma)=\mathrm{d} f \bar{\otimes} \sigma+f D(\sigma)$ means that $f \sigma$ cannot be holomorphic unless $\bar{\partial} f=0$ which, as explained in Section 4 (at least for generic $q$ ), means $f$ a multiple of 1 . This reminds us that $\mathcal{E}_{-n}^{\text {hol }}$ is indeed a complex vector space but not a $\mathbb{C}_{q}\left[S^{2}\right]$-module. As such we have seen that it contains $\operatorname{span}\left\{c^{s} a^{t}\right\}$, which are linearly independent over $\mathbb{C}$ and give the usual $n+1$-dimensional corepresentation. On the other hand, since the dimension of $\mathcal{E}_{-n}^{\text {hol }}$ classically is $n+1$, this should also be true for generic $q$ (since all our structures deform with classical dimensions). In this case, by dimensions, $\mathcal{E}_{-n}^{\text {hol }}=\operatorname{span}\left\{c^{s} a^{t}\right\}$ i.e. not only contains but coincides with the $q$-deformed $n+1$-dimensional corepresentation of $\mathbb{C}_{q}\left[S L_{2}\right]$. This outlines a geometric proof of the $q$-Borel-Weil-Bott construction.

\section{REFERENCES}

[BCT] F. Bonechi, N. Ciccoli and M. Tarlini. Noncommutative instantons in the 4-sphere from quantum groups. Commun. Math. Phys., 226:419-432, 2002.

[BM1] T. Brzeziński and S. Majid. Quantum group gauge theory on quantum spaces. Commun. Math. Phys., 157:591-638, 1993. Erratum 167:235, 1995.

[BM2] T. Brzeziński and S. Majid. Quantum differentials and the $q$-monopole revisited. Acta Appl. Math., 54:185-232, 1998.

[Co] A. Connes. Noncommutative Geometry. Academic Press, 1994.

$[\mathrm{HM}]$ P. Hajac and S. Majid. Projective module description of the $q$-monopole. Commun. Math. Phys., 206:246-464, 1999.

[Ma] S. Majid. Foundations of Quantum Group Theory. Cambridge Univeristy Press, 1995.

[M1] S. Majid. Quantum and braided group Riemannian geometry. J. Geom. Phys., 30:113-146, 1999.

[M2] S. Majid. Riemannian Geometry of Quantum Groups and Finite Groups with Nonuniversal Differentials. Commun. Math. Phys. 225:131-170 (2002).

[M3] S. Majid. Ricci tensor and Dirac operator on $\mathbb{C}_{q}\left[S L_{2}\right]$ at roots of unity. Lett. Math. Phys., 63:39-54, 2003.

[PS] A. Pinzul and A. Stern. Dirac operator on the quantum sphere. Phys. Lett. B 512:217-224, 2001.

[Po1] P. Podles. Quantum spheres. Lett. Math. Phys., 14:193-202, 1987.

[Po2] P. Podles. Differential calculus on quantum spheres. Lett. Math. Phys., 18:107-119, 1989.

[Po3] P. Podles. The classification of differentical structures on quantum 2-spheres. Commun. Math. Phys. 150: 167-179, 1992.

[Sw] M. Sweedler Hopf Algebras. Benjamin Press, 1969. 
[Sch] H-J. Schneider. Principal homogeneous spaces for arbitrary Hopf algebras. Isr. J. Math., 72:167-195, 1990.

[Wo] S.L. Woronowicz. Differential calculus on compact matrix pseudogroups (quantum groups). Commun. Math. Phys., 122:125-170, 1989.

[GZ] A.R. Gover and R.B. Zhang. Geometry of quantum homogeneous vector bundles and representation theory of quantum groups I (appendix of), q-alg/9705016 and Review Math. Phys. 11 (1999) 533-552.

School of Mathematical Sciences, Queen Mary, University of London, 327 Mile End RD, LONDON E1 4NS, UK 